\title{
Proximal and distal factors predicting timely initiation of breast feeding in Ethiopia: a systematic review and meta-analysis
}

Tesfa Dejenie Habtewold ${ }^{1,2^{*}}$, Shimels Hussien Mohammed ${ }^{3}$, Aklilu Endalamaw ${ }^{4}$, Henok Mulugeta ${ }^{5}$, Getenet Dessie ${ }^{6}$, Derbew Fikadu Berhe ${ }^{7}$, Mulugeta Molla Birhanu ${ }^{8}$, Md. Atiqul Islam $^{9}$, Andreas A. Teferra ${ }^{10}$, Nigus Gebremedhin Asefa ${ }^{2}$, Sisay Mulugeta Alemu ${ }^{11}$

${ }^{1}$ Department of Nursing, College of Medicine and Health Science, Debre Berhan University, Debre Berhan, Ethiopia

${ }^{2}$ Department of Epidemiology, University Medical Centre Groningen, University of Groningen, Groningen, The Netherlands

${ }^{3}$ Department of Community Nutrition, School of Nutritional Sciences and Dietetics, Tehran University of Medical Sciences-International Campus (TUMS-IC), Tehran, the Islamic Republic of Iran

${ }^{4}$ Department of Pediatrics and Child Health Nursing, School of Nursing, College of Medicine and Health Sciences, University of Gondar, Gondar, Ethiopia.

${ }^{5}$ Department of Nursing, College of Health Science, Debre Markos University, Debre Markos, Ethiopia

${ }^{6}$ Department of Nursing, School of Health Science, College of Medicine and Health Science, Bahir Dar University, Bahir Dar, Ethiopia

${ }^{7}$ School of Pharmacy, College of Health Science, Mekelle University, Mekelle, Ethiopia

${ }^{8}$ School of Clinical Sciences, Department of Medicine, Epidemiology and Prevention Division, Monash University, Melbourne, Australia

${ }^{9}$ Department of Statistics, Shahjalal University of Science and Technology, Sylhet, Bangladesh

${ }^{10}$ Division of Epidemiology, College of Public Health, The Ohio State University, Columbus, $\mathrm{OH}$, USA

${ }^{11}$ Department of Public Health, University Medical Centre Groningen, University of Groningen, Groningen, The Netherlands

* =Corresponding author

Tesfa Dejenie Habtewold

Email: tesfadej2003@gmail.com 
medRxiv preprint doi: https://doi.org/10.1101/19000497; this version posted July 8, 2019. The copyright holder for this preprint (which was not certified by peer review) is the author/funder, who has granted medRxiv a license to display the preprint in perpetuity.

It is made available under a CC-BY-NC-ND 4.0 International license .

\begin{abstract}
Background: In Ethiopia, the current coverage of timely initiation of breast feeding (TIBF) has fallen short of the national Health Sector Transformation Plan 2016-2020, National Nutrition Program 2016-2020 and WHO global target. This calls for the need to assess relevant proximal and distal factors that affect the rate of TIBF in Ethiopia.
\end{abstract}

Objective: The aim of this meta-analysis was to investigate the association between TIBF and educational status, household income, marital status, media exposure, and parity in Ethiopia.

Methods: Databases used were PubMed, EMBASE, Web of Science, SCOPUS, CINAHL and WHO Global health library, and key terms were searched using interactive searching syntax. It was also supplemented by manual searching. Observational studies published between September 2000 and March 2019 were included. The methodological quality of studies was examined using the Newcastle-Ottawa Scale (NOS) for cross-sectional studies. Data were extracted using the Joanna Briggs Institute (JBI) data extraction tool. To obtain the pooled odds ratio (OR), extracted data were fitted in a random-effects meta-analysis model. Statistical heterogeneity was quantified using Cochran's $Q$ test, $\mathrm{\tau}^{2}$, and $\mathrm{I}^{2}$ statistics. Additional analysis conducted includes Jackknife sensitivity analysis, cumulative metaanalysis, and meta-regression analysis.

Results: Out of 553 studies retrieved, 25 studies fulfilled our inclusion criteria. Almost all studies were conducted on mothers with newborn less than 23 months. Maternal educational status (OR $\left.=1.82 ; \mathrm{p}<0.001 ; 95 \% \mathrm{Cl}=1.35-2.45 ; \mathrm{I}^{2}=84.96 \%\right)$, paternal educational status (OR $=2.72 ; p=0.001,95 \% \mathrm{Cl}=1.49-4.97 \mathrm{I}^{2}=62.50 \%$ ), income (OR = $\left.1.16 ; p=0.002 ; 95 \% \mathrm{Cl}=1.05-1.27 ; I^{2}=0.00 \%\right)$, marital status $(\mathrm{OR}=1.39 ; \mathrm{p}=0.001 ; 95 \% \mathrm{Cl}$ $\left.=1.14-1.69 ; I^{2}=9.17 \%\right)$ and parity $\left(O R=1.39 ; p=0.01 ; 95 \% \mathrm{Cl}=1.07-1.81 ; I^{2}=74.43 \%\right)$ were found to be significantly associated with TIBF. We also observed a direct doseresponse relationship of TIBF with educational status and income.

Conclusions: Proximal and distal factors significantly predicting TIBF practice in Ethiopia, which needs integrated intervention by health professionals and healthcare policymakers. Health education, counselling and peer education targeting parents at antenatal and postnatal periods are needed. It is also relevant to improve the economic power of women and promote gender equality. 
medRxiv preprint doi: https://doi.org/10.1101/19000497; this version posted July 8, 2019. The copyright holder for this preprint (which was not certified by peer review) is the author/funder, who has granted medRxiv a license to display the preprint in perpetuity.

It is made available under a CC-BY-NC-ND 4.0 International license .

\section{Background}

Timely initiation of breast feeding (TIBF) is defined as putting the newborn to the breast within the first-hour birth. $(1,2)$ Based on the recent UNICEF and World Health Organization (WHO) global report, approximately 78 million newborns are not breastfeed within the first hour after birth; majority are born in low- and middle-income countries (LMICs).(3) Breast feeding with timely initiation and optimal duration is a core component of primary healthcare that boosts newborn later life health (e.g. cognitive function)(4), reduce infection, and prevent up to $60 \%$ infant and child morbidity and mortality related to malnutrition.(5-11) Recent estimates suggest that optimal breast feeding could prevent approximately $12 \%$ deaths per year in under-five children, which represents around 800,000 lives in LMICs.(12) Likewise, TIBF has been shown to reduce neonatal mortality by $22 \%$.(7) Breast feeding also prevents acute and chronic maternal diseases. $(13,14)$ Optimal breast feeding has also economic and environmental advantages.(13)

Suboptimal breast feeding including delayed initiation increases risk of newborn morbidity and mortality up to fivefold.(5, 15-17) The 2010 Global Burden of Disease study reveals that suboptimal breast feeding is one of the three leading causes of disease in SubSaharan African (SSA) countries.(18) Approximately 25 to $50 \%$ of infant deaths in developing countries occur due to poor breast feeding practice. $(16,19)$ In terms of the global absolute number of under-five child deaths, Ethiopia ranked sixth with about 472,000 deaths per year. The age distribution of under-five deaths varies from $29 \%$ in the first 30 days of life to $42 \%$ in the fourth year.(20) Neonatal mortality rate is still high in Ethiopia, which accounts for $42 \%$ of under-five deaths. $(21,22)$ Based on our previous meta-analysis of 13 observational studies conducted in Ethiopia, the prevalence of neonatal mortality is $16.3 \% .(23)$

To date, only 22 countries achieved WHO target of 70\% coverage in TIBF.(24) Although LMICs have strong breast feeding traditions compared to developed countries, adherence to $\mathrm{WHO}$ recommendations of TIBF is poor with considerable difference between nations. A report from 76 countries on the rates and trends of TIBF over the past decade shows that TIBF rates varied widely across regions from 32\% in East Asia and the Pacific to 65\% in Eastern and Southern Africa.(25) A demographic and health survey conducted in 57 LMICs involving nearly 200,000 newborn shows that $39 \%$ of newborn (with regional range 
medRxiv preprint doi: https://doi.org/10.1101/19000497; this version posted July 8, 2019. The copyright holder for this preprint (which was not certified by peer review) is the author/funder, who has granted medRxiv a license to display the preprint in perpetuity. It is made available under a CC-BY-NC-ND 4.0 International license .

from 31 to $60 \%$ ) breastfed within one hour of birth.(26) Likewise, a report of demographic and health surveys conducted since 2010 in 32 SSA countries show that the prevalence of TIBF is 50.5\%.(27) A systematic review of studies conducted between 1999 and 2013 in Asia, Africa, and South America reveal the prevalence of TIBF ranges from $11.4 \%$ in a province of Saudi Arabia to $83.3 \%$ in Sri Lanka.(28) In Ethiopia, breast feeding is a common practice with $96 \%$ of mothers breastfeed at least once during their lifetime.(29) According to our previous meta-analysis of 45 observational studies, the prevalence of TIBF in Ethiopia is 66.5\%.(30)

Numerous country-specific or global factors influence mothers' decision and ability to initiate breast feeding within one hour of birth. The accumulated body of evidence from regional and global studies consistently shows that TIBF has been associated with numerous factors including educational status, household income, marital status, media exposure and parity.(31-39) In our previous systematic review of 70 observational studies, we identified 18 predictors categorized into four groups: proximal (maternal occupational status, maternal knowledge on TIBF, and breast feeding guidance and counselling), proximalintermediate (place of delivery, mode of delivery, birth attendant and sex of newborn), distal-intermediate (antenatal care, postnatal care, prelacteal feeding, and colostrum feeding) and distal (paternal educational status, household income, marital status, media exposure, family size, breast feeding experience, place of residence, birth order, parity, and iron-folate supplementation).(30) In the follow-up meta-analyses(30,40,41), we confirmed that breast feeding guidance and counselling, mode of delivery, place of delivery, antenatal care visit and colostrum discarding significantly associated with TIBF practice, whereas maternal occupational status, maternal or caregiver's age and sex of newborn not significantly associated with TIBF.

Given the multidimensional benefits of breast feeding for the newborn, mother and community, global initiatives have been made to improve the national and international breast feeding practice. Some of these initiatives are the International Code of Marketing of Breast-milk Substitutes (aka the Code), Innocenti Declaration, Baby Friendly Hospital Initiative (BFHI) (i.e. Ten Steps to Successful Breast feeding), Millennium Development Goals, Global Nutrition Targets 2025 and Sustainable Development Goals. $(31,42)$ The Ethiopian government has endorsed and implemented these aforementioned policies and programs to reduce infant and child mortality and morbidity related to poor breast feeding 
in the country. Since 2004, the Ethiopian Federal Ministry of Health has developed infant and young child feeding guidelines and provided breast feeding promotions.(43) A national nutrition strategy and program (NNP) has also been developed and implemented in a multisectoral approach. To improve the nutritional status of mothers and children, the Health Sector Development Plan (HSDP)-IV has integrated nutrition into the health extension program.(44)

Consequently, the rate of malnutrition, stunting, underweight and under-five mortality have declined in Ethiopia.(45) Despite the heartening reduction of under-five morbidity and mortality, the rate of TIBF has fallen short of the national Health Sector Transformation Plan 2016-2020(44), National Nutrition Program 2016-2020(46) and WHO global target.(24) This calls for the need to investigate the pooled effect of various proximal and distal factors predicting TIBF. Therefore, the aim of this meta-analysis was to investigate the association between TIBF and maternal educational status, paternal educational status, household income, marital status, media exposure, and parity in Ethiopia. We hypothesized high educational status, high household income, being married, exposed to media and multiparity significantly increase the odds of TIBF. 
medRxiv preprint doi: https://doi.org/10.1101/19000497; this version posted July 8, 2019. The copyright holder for this preprint (which was not certified by peer review) is the author/funder, who has granted medRxiv a license to display the preprint in perpetuity. It is made available under a CC-BY-NC-ND 4.0 International license .

\section{Methods}

\section{Measurements}

The outcome measurement was timely initiation of breast feeding (TIBF), which is the proportion of children born in the last two years who were breastfeed within the first hour of delivery.(47) Maternal educational status, paternal educational status, household income, marital status, media exposure, and parity were selected predicting factors. The selection of these factors was guided by our previous systematic review findings.(30) Educational status represents the highest schooling grade achieved as per the Ethiopian educational system and categorized as 'uneducated' (including these who able to read and write without formal schooling), 'primary' (grades 1 to 8) and 'secondary and above' (grades 9 or above). Household income was categorized as 'high', 'medium' and 'low'. Because of substantial inconsistency in the reported household income, we used a harmonized qualitative way of classification of income for all included studies that reported at least three categories of household income or wealth index based on authors educational judgment. Marital status was categorized as 'currently married' and 'others' (i.e. single, divorced, widowed). Media exposure represents exposure to or ownership of any print media (newspaper, leaflet, brochure) and broadcasting (radio and television) and categorized as 'yes' and 'no'. This does not include Facebook, email, YouTube, or WhatsApp as most mothers do not have access to internet. If studies reported accessibility or exposure to more than one media tools, we extracted data on radio (as 'yes') followed by television and print media. Parity refers to the total number of births after 28 weeks and was categorized as 'primipara' if the mothers have only one birth and 'multipara' if the mothers have at least two births.

\section{Search for literature}

Databases searched were PubMed, EMBASE, Web of Science, SCOPUS, CINAHL and WHO Global health library. The interactive searching syntax was developed for all databases in consultation with librarian, experts on literature searching (Supplementary file 1). We further manually searched the table of contents of Ethiopian Journal of Health Development, Ethiopian Journal of Health Sciences, Ethiopian Journal of Reproductive Health, International Breast feeding Journal, BMC Pregnancy and Childbirth, BMC Public 
medRxiv preprint doi: https://doi.org/10.1101/19000497; this version posted July 8, 2019. The copyright holder for this preprint (which was not certified by peer review) is the author/funder, who has granted medRxiv a license to display the preprint in perpetuity. It is made available under a CC-BY-NC-ND 4.0 International license .

Health, BMC Paediatrics, Nutrition Journal and Italian Journal of Paediatrics. We also searched cross-references and grey literature on Addis Ababa University institutional research collection repository database. The last search was done in March 2019.

\section{Inclusion and exclusion criteria}

The studies were included when they met the following inclusion criteria: (1) observational studies, such as cross-sectional, case-control and cohort studies; (2) studies conducted in Ethiopia; (3) studies reported on the association between TIBF (i.e. operationalized based on the WHO definition) and maternal and paternal educational status, household income (at least three categories must be reported), marital status, media exposure (not exposed or no access to media category must be reported) and parity; (4) studies published from September 2000 (i.e. the time when last revision of the global breast feeding recommendations occurred) to March 2019. Program evaluation reports, systematic reviews and meta-analyses, qualitative studies and studies on mothers with medical conditions including HIV/AIDS and pre-term or ill health newborn were excluded.

\section{Screening and selection of studies}

Initially, all identified studies were exported into RefWorks citation manager version 4.6 for Windows. Afterward, duplicate studies were deleted from further screening. Next, a pair of reviewers independently screened the abstracts and titles using Microsoft Excel spreadsheet for relevance, their compliance with our measurements of interest and against our inclusion criteria. Based on Cohen's Kappa inter-rater reliability test, the agreement between the two reviewers was 0.76 indicating substantial agreement between the two reviewers. Disagreements on the inclusion of titles or abstracts were solved through discussion and consensus. After removing irrelevant studies, full text of selected abstracts were downloaded and reviewed for further eligibility. PRISMA flow diagram was also used to illustrate the screening and selection processes of studies.(48) Finally, two independent reviewers (TD and SM) extracted the following information from each included studies using Joanna Briggs Institute (JBI) data extraction tool(49): author name, publication year, residence, study design, study population, number of participants, source of funding, and observed data. If funding source was not explicitly mentioned, we reported as 'not mentioned', whereas 'no funding' category was used if the author explicitly mentioned 
medRxiv preprint doi: https://doi.org/10.1101/19000497; this version posted July 8, 2019. The copyright holder for this preprint (which was not certified by peer review) is the author/funder, who has granted medRxiv a license to display the preprint in perpetuity. It is made available under a CC-BY-NC-ND 4.0 International license .

there is no funding or funding not applicable. If funding was not given directly but through other donors, the original donor was mentioned. Newcastle-Ottawa Scale (NOS) for crosssectional studies was used to examine the quality of studies and the potential risk of bias.(50) The scoring system, selection of cut-off value and interpretation used in this metaanalysis were published in our protocol.(51) Furthermore, we reported the results in compliance with the recommendation of Preferred Reporting Items for Systematic review and Meta-analysis (PRISMA) statement (Supplementary file 2).(48)

\section{Statistical analysis}

To obtain the pooled odds ratio (OR), extracted data were fitted in a random-effects metaanalysis model. In addition, a cumulative meta-analysis was done to illustrate the trend of evidence regarding the effect of predictors on TIBF and interpreted as stable, steadily increased/decreased, slightly increased/decreased or dramatically increased/decreased. Publication bias was assessed by subjective evaluation of the funnel plot, and then, we performed Egger's regression statistical test to objectively confirm the presence of significant publication bias at $p$-value $\leq 0.01$.(52) We used Cochran's $Q$ test to test heterogeneity, $\tau^{2}$ to estimate the amount of total/residual between-study variance, and $\mathrm{I}^{2}$ statistics to measure the proportion of total variation between study due to heterogeneity.(53) Clinical and methodology heterogeneity were also carefully evaluated. Factors attributed to between-study heterogeneity were investigated using mixed-effects meta-regression analysis using region, residence, sample size and publication year as covariates.(54) The residual amount of heterogeneity was subtracted from the proportion of heterogeneity and divided by the total amount of heterogeneity to obtain the total amount of heterogeneity $\left(R^{2}\right)$ explained by covariates. Omnibus test of moderators was applied to assess the moderation effect of these covariates. Meta-regression analysis was done only when heterogeneity threshold $\left(1^{2}\right)$ was $\geq 80 \%$. Jackknife sensitivity analysis was done to examine the influence of outlier studies on the pooled OR estimate, a significance level of estimate and between-study heterogeneity.(55) The study was excluded when the pooled OR estimate increased or decreased by one and changes the significance level after lifting out that study from the meta-analysis. Because of the small number of studies available for some variables, the change in heterogeneity threshold was not considered as a primary 
criterion to detect and excluded the outlier study. The data were analyzed using "metafor" packages in R software version 3.2.1 for Windows.(54)

\section{Review protocol}

This systematic review and meta-analysis was conducted based on the registered (CRD42017056768) and published protocol.(51) Based on the authors' decision, the following changes were made to the published protocol.(51) Joanna Briggs Institute (JBI) tool(49) was used to extract the relevant data. Furthermore, cumulative meta-analysis and mixed-effects meta-regression analysis were done to reveal the trends of evidence and identify possible sources of between-study heterogeneity respectively. 


\section{Results}

\section{Search results}

In total, 483 studies through electronic databases and 70 studies through manual searching were retrieved for further screening. Following a rigorous screening, 28 studies were selected for full text review. Three studies were excluded after the full text review: one study(56) reported only the prevalence of TIBF, and the other two studies(20,57) did not report the selected factors of our interest. A total of 25 studies were included in this metaanalysis, which most of them conducted on mothers with newborn less than 23 months. All studies had good methodological quality (NOS score $\geq 7$ ). One study reported more than one predicting factors. The screening and selection process are illustrated below using the PRISMA flow diagram (Figure 1). The detailed characteristics of studies that reported each variable is presented in Table 1. 
medRxiv preprint doi: https://doi.org/10.1101/19000497; this version posted July 8, 2019. The copyright holder for this preprint (which was not certified by peer review) is the author/funder, who has granted medRxiv a license to display the preprint in perpetuity.

It is made available under a CC-BY-NC-ND 4.0 International license .

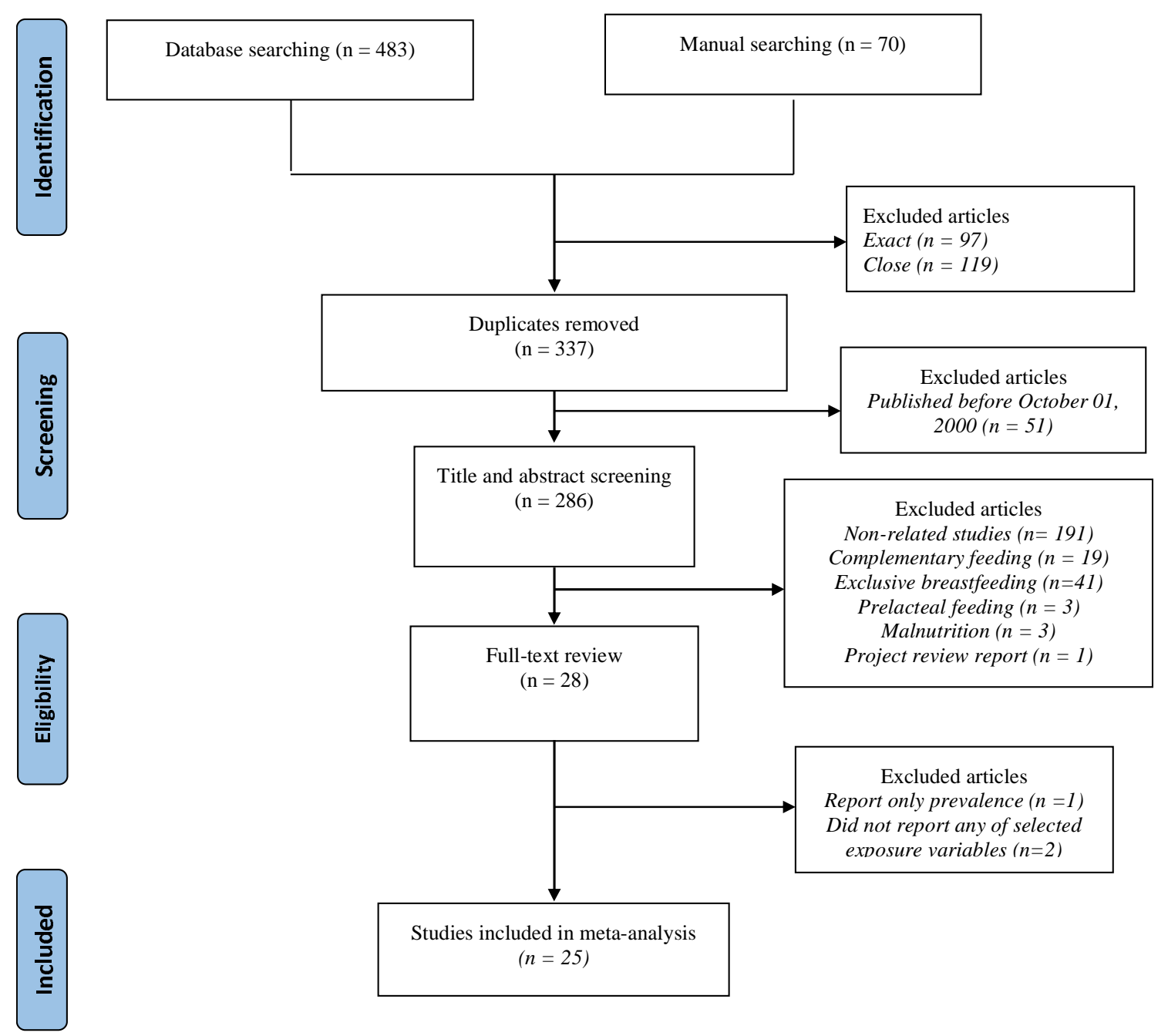

Figure 1. PRISMA flow diagram showing the schematics of literature screening and selection. 
Table 1: Detailed characteristics of studies that reported each variable of interest.

\begin{tabular}{|c|c|c|c|c|c|c|c|c|c|c|}
\hline \multirow[t]{2}{*}{ Study } & \multirow[t]{2}{*}{ Region } & \multirow[t]{2}{*}{ Study design } & \multirow[t]{2}{*}{ Study population } & \multirow{2}{*}{$\begin{array}{l}\text { Sample size/ } \\
\text { Participated }\end{array}$} & \multirow[t]{2}{*}{ Funding source } & \multirow[t]{2}{*}{ Predicting factors } & \multicolumn{3}{|c|}{ TIBF } & \multirow{3}{*}{ 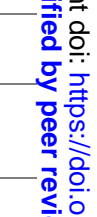 } \\
\hline & & & & & & & $\begin{array}{l}\text { Within } \\
1 \text { hour }\end{array}$ & $\begin{array}{l}\text { After } 1 \\
\text { hour }\end{array}$ & Total & \\
\hline \multicolumn{7}{|c|}{ a. Maternal educational status } & & & & \\
\hline \multirow{4}{*}{$\begin{array}{l}\text { Adugna et.al } \\
2014(58)\end{array}$} & \multirow{4}{*}{$\begin{array}{l}\text { SNNPR, Arba } \\
\text { Minch Zuria }\end{array}$} & \multirow{4}{*}{$\begin{array}{l}\text { Cross-sectional } \\
\text { study }\end{array}$} & \multirow{4}{*}{$\begin{array}{l}\text { Women who had children } \\
\text { under two years }\end{array}$} & \multirow[t]{4}{*}{$384 / 383$} & \multirow{4}{*}{$\begin{array}{l}\text { Arba Minch } \\
\text { University }\end{array}$} & Uneducated & 172 & 66 & 238 & \\
\hline & & & & & & Primary & 130 & 18 & 148 & \\
\hline & & & & & & Secondary or above & 147 & 15 & 162 & \\
\hline & & & & & & Total & 449 & 99 & 548 & \\
\hline \multirow[t]{4}{*}{ Gultie et.al 2016(59) } & \multirow{4}{*}{$\begin{array}{l}\text { Amhara, Debre } \\
\text { Berhan town }\end{array}$} & \multirow{4}{*}{$\begin{array}{l}\text { Cross-sectional } \\
\text { study }\end{array}$} & \multirow{4}{*}{$\begin{array}{l}\text { Mothers having children } \\
\text { aged less than } 23 \text { months } \\
\text { old }\end{array}$} & \multirow[t]{4}{*}{$548 / 548$} & \multirow[t]{4}{*}{ Not mentioned } & Uneducated & 172 & 66 & 238 & \\
\hline & & & & & & Primary & 130 & 18 & 148 & \\
\hline & & & & & & Secondary or above & 147 & 15 & 162 & \\
\hline & & & & & & Total & 449 & 99 & 548 & \\
\hline \multirow[t]{4}{*}{ Derso et.al $2017(60)^{*}$} & \multirow{4}{*}{$\begin{array}{l}\text { Amhara, Dabat } \\
\text { district }\end{array}$} & \multirow{4}{*}{$\begin{array}{l}\text { Cross-sectional } \\
\text { study }\end{array}$} & \multirow{4}{*}{$\begin{array}{l}\text { Mothers with children } \\
\text { under five years of age }\end{array}$} & \multirow[t]{4}{*}{$6,761 / 6,761$} & \multirow[t]{4}{*}{ PEPFAR-CDC } & Uneducated & 1767 & 2607 & 4374 & \\
\hline & & & & & & Primary & 663 & 875 & 1538 & \\
\hline & & & & & & Secondary or above & 539 & 310 & 849 & \\
\hline & & & & & & Total & 2969 & 3792 & 6761 & 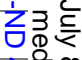 \\
\hline \multirow[t]{4}{*}{ John et al 2019(61)* } & \multirow[t]{4}{*}{ National } & Cross-sectional & Mothers with children less & $5,546 / 5,546$ & No funding & Uneducated & 1851 & 636 & 2487 & \\
\hline & & & than 24 months of age & & & Primary & 955 & 318 & 1273 & \\
\hline & & & & & & Secondary or above & 258 & 103 & 361 & \\
\hline & & & & & & Total & 3064 & 1057 & 4121 & \\
\hline Ekubay et al 2018(62) & Addis Ababa & Cross-sectional & mothers with infants & $597 / 583$ & Addis Ababa & Uneducated & 38 & 28 & 66 & \\
\hline & Town & study & younger than or equal to six & & University & Primary & 73 & 66 & 139 & \\
\hline & & & months of age & & & Secondary or above & 218 & 141 & 359 & \\
\hline & & & & & & Total & 329 & 235 & 564 & \\
\hline Alemayehu et.al. & Tigray, Axum & Cross-sectional & Mothers who had children & $418 / 418$ & Mekelle University & Uneducated & 27 & 21 & 48 & \\
\hline $2014(63)$ & town & study & aged 6 to 12 & & & Primary & 128 & 89 & 217 & \\
\hline & & & months & & & Secondary or above & 92 & 64 & 156 & \\
\hline & & & & & & Total & 247 & 174 & 421 & \\
\hline Berhe et.al. 2013(64) & Tigray, Mekelle & Cross-sectional & Mothers of children aged 0 & $361 / 361$ & Addis Ababa & Uneducated & 85 & 30 & 115 & \\
\hline
\end{tabular}




\begin{tabular}{|c|c|c|c|c|c|c|c|c|c|c|}
\hline & \multirow[t]{3}{*}{ town } & \multirow[t]{3}{*}{ study } & \multirow[t]{3}{*}{ to 24 months } & & \multirow[t]{3}{*}{ University } & Primary & 64 & 22 & 86 & 요용. \\
\hline & & & & & & Secondary or above & 129 & 27 & 156 & \\
\hline & & & & & & Total & 278 & 79 & 357 & \\
\hline \multirow{4}{*}{$\begin{array}{l}\text { Beyene et.al. } \\
2017(65)\end{array}$} & \multirow{4}{*}{$\begin{array}{l}\text { SNNPR, Dale } \\
\text { Woreda }\end{array}$} & \multirow{4}{*}{$\begin{array}{l}\text { Cross-sectional } \\
\text { study }\end{array}$} & \multirow{4}{*}{$\begin{array}{l}\text { Mothers of children under } \\
24 \text { months }\end{array}$} & \multirow[t]{4}{*}{$634 / 634$} & \multirow{4}{*}{$\begin{array}{l}\text { Addis Ababa } \\
\text { University }\end{array}$} & Uneducated & 129 & 14 & 143 & \\
\hline & & & & & & Primary & 388 & 87 & 475 & \\
\hline & & & & & & Secondary or above & - & - & - & \\
\hline & & & & & & Total & 517 & 101 & 618 & \\
\hline \multirow{4}{*}{$\begin{array}{l}\text { Lakew et.al. } \\
2015(66)^{*}\end{array}$} & \multirow[t]{4}{*}{ National } & \multirow{4}{*}{$\begin{array}{l}\text { Cross-sectional } \\
\text { study }\end{array}$} & \multirow{4}{*}{$\begin{array}{l}\text { Mothers who had children } \\
\text { less than } 5 \text { years }\end{array}$} & \multirow[t]{4}{*}{$11,654 / 11,553$} & \multirow[t]{4}{*}{ Not mentioned } & Uneducated & 4244 & 3779 & 8023 & \\
\hline & & & & & & Primary & 1677 & 1434 & 3111 & \\
\hline & & & & & & Secondary or above & 259 & 160 & 419 & \\
\hline & & & & & & Total & 6180 & 5373 & 11553 & \\
\hline \multirow[t]{4}{*}{ Liben et.al. 2016(67) } & \multirow{4}{*}{$\begin{array}{l}\text { Afar, Dubti } \\
\text { town }\end{array}$} & \multirow{4}{*}{$\begin{array}{l}\text { Cross-sectional } \\
\text { study }\end{array}$} & \multirow{4}{*}{$\begin{array}{l}\text { Mothers of infants aged less } \\
\text { than } 6 \text { months }\end{array}$} & \multirow[t]{4}{*}{$346 / 333$} & \multirow[t]{4}{*}{ Samara University } & Uneducated & 35 & 87 & 122 & \\
\hline & & & & & & Primary & 116 & 143 & 259 & \\
\hline & & & & & & Secondary or above & - & - & - & \\
\hline & & & & & & Total & 151 & 230 & 381 & กิ⿻ \\
\hline \multirow{4}{*}{$\begin{array}{l}\text { Setegn et.al. } \\
2011(68)\end{array}$} & \multirow{4}{*}{$\begin{array}{l}\text { Oromia, Goba } \\
\text { district }\end{array}$} & \multirow{4}{*}{$\begin{array}{l}\text { Cross-sectional } \\
\text { study }\end{array}$} & \multirow{4}{*}{$\begin{array}{l}\text { Mothers with children less } \\
\text { than } 12 \text { months }\end{array}$} & \multirow[t]{4}{*}{$668 / 608$} & \multirow[t]{4}{*}{ Jimma University } & Uneducated & 111 & 122 & 233 & 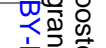 \\
\hline & & & & & & Primary & 148 & 123 & 271 & 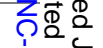 \\
\hline & & & & & & Secondary or above & 61 & 34 & 95 & Z㤀 \\
\hline & & & & & & Total & 320 & 279 & 599 & 获员 \\
\hline Mekonen et.al. & Amhara, & Cross-sectional & Mothers of infants & $845 / 823$ & Not mentioned & Uneducated & 149 & 185 & 334 & \\
\hline & South Gondar & study & under 12 months & & & Primary & 252 & 237 & 489 & 包 \\
\hline & & & & & & Secondary or above & - & - & - & 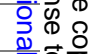 \\
\hline & & & & & & Total & 401 & 422 & 823 & $\overline{\overline{\bar{\sigma}}} \div$ \\
\hline Tamiru et.al 2012(70) & Oromia, Jimma & Cross-sectional & Mothers of index children & $384 / 382$ & Not mentioned & Uneducated & 191 & 122 & 313 & $\frac{\mathbb{D}}{0} \frac{0}{0}$ \\
\hline & Arjo Woreda & study & aged 0 to 6 months & & & Primary & 22 & 18 & 40 & \\
\hline & & & & & & Secondary or above & - & - & - & $\frac{\pi}{2}$ \\
\hline & & & & & & Total & 213 & 140 & 353 & 을. 을 \\
\hline Tamiru et.al 2015(71) & SNNPR, Arba & Cross-sectional & Mothers of infants aged two & $384 / 384$ & Arba & Uneducated & 172 & 142 & 314 & \\
\hline & Minch Zuria & study & years and younger & & Minch University & Primary & 32 & 18 & 50 & \\
\hline & Woreda & & & & & Secondary or above & 16 & 4 & 20 & $\stackrel{\Phi}{\stackrel{\Phi}{口}}$ \\
\hline & & & & & & Total & 220 & 164 & 384 & $₹$ \\
\hline
\end{tabular}

\section{Page 13 of 38}




\begin{tabular}{|c|c|c|c|c|c|c|c|c|c|c|}
\hline \multirow{4}{*}{$\begin{array}{l}\text { Deregh et al } \\
2012(72)\end{array}$} & \multirow[t]{4}{*}{ Addis Ababa } & \multirow{4}{*}{$\begin{array}{l}\text { Cross-sectional } \\
\text { study }\end{array}$} & \multirow{4}{*}{$\begin{array}{l}\text { Mothers of babies admitted } \\
\text { to the Neonatal Intensive } \\
\text { Care Unit }\end{array}$} & \multirow[t]{4}{*}{$429 / 429$} & \multirow[t]{4}{*}{ Not mentioned } & Uneducated & 7 & 37 & 44 & \\
\hline & & & & & & Primary & 18 & 78 & 96 & \\
\hline & & & & & & Secondary or above & 34 & 166 & 200 & \\
\hline & & & & & & Total & 59 & 281 & 340 & \\
\hline \multirow{4}{*}{$\begin{array}{l}\text { Woldemichael et.al } \\
2016(73)\end{array}$} & \multirow{4}{*}{$\begin{array}{l}\text { Oromia, Tiyo } \\
\text { Woreda }\end{array}$} & \multirow{4}{*}{$\begin{array}{l}\text { Cross-sectional } \\
\text { study }\end{array}$} & \multirow{4}{*}{$\begin{array}{l}\text { Mothers who have children } \\
\text { less than one-year age }\end{array}$} & \multirow[t]{4}{*}{$386 / 373$} & \multirow{4}{*}{$\begin{array}{l}\text { Addis Ababa } \\
\text { University }\end{array}$} & Uneducated & 63 & 57 & 120 & \\
\hline & & & & & & Primary & 133 & 53 & 186 & \\
\hline & & & & & & Secondary or above & 55 & 12 & 67 & \\
\hline & & & & & & Total & 251 & 122 & 373 & \\
\hline \multirow[t]{4}{*}{ Tamir 2010(74) } & \multirow{4}{*}{$\begin{array}{l}\text { Amhara, Bahir } \\
\text { Dar city }\end{array}$} & \multirow{4}{*}{$\begin{array}{l}\text { Cross-sectional } \\
\text { study }\end{array}$} & \multirow{4}{*}{$\begin{array}{l}\text { Mothers having children 0- } \\
23 \text { months of age }\end{array}$} & \multirow[t]{4}{*}{$856 / 825$} & \multirow{4}{*}{$\begin{array}{l}\text { Addis Ababa } \\
\text { University }\end{array}$} & Uneducated & 79 & 35 & 114 & 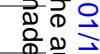 \\
\hline & & & & & & Primary & 132 & 49 & 181 & $\stackrel{0}{\aleph}$ \\
\hline & & & & & & Secondary or above & 419 & 71 & 490 & 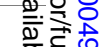 \\
\hline & & & & & & Total & 630 & 155 & 785 & \\
\hline \multicolumn{10}{|c|}{ b. Paternal educational status } & $\frac{2}{0} \leq$ \\
\hline \multirow[t]{4}{*}{ John et al $2019(61)^{*}$} & \multirow[t]{4}{*}{ National } & \multirow{4}{*}{$\begin{array}{l}\text { Cross-sectional } \\
\text { study }\end{array}$} & \multirow{4}{*}{$\begin{array}{l}\text { Mothers with children less } \\
\text { than } 24 \text { months of age }\end{array}$} & \multirow[t]{4}{*}{$5,546 / 5,546$} & \multirow[t]{4}{*}{ No funding } & Uneducated & 1285 & 470 & 1755 & ब \\
\hline & & & & & & Primary & 1221 & 356 & 1577 & مై \\
\hline & & & & & & Secondary or above & 416 & 152 & 568 & ج) \\
\hline & & & & & & Total & 2922 & 978 & 3900 & గุ̣. \\
\hline \multirow[t]{4}{*}{ Tamiru et.al 2015(71) } & \multirow{4}{*}{$\begin{array}{l}\text { SNNPR, Arba } \\
\text { Minch Zuria } \\
\text { Woreda }\end{array}$} & \multirow{4}{*}{$\begin{array}{l}\text { Cross-sectional } \\
\text { study }\end{array}$} & Mothers of infants aged two & $384 / 384$ & Arba & Uneducated & 172 & 142 & 314 & 吂岢宅 \\
\hline & & & years and younger & & Minch University & Primary & 32 & 18 & 50 & 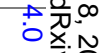 \\
\hline & & & & & & Secondary or above & 16 & 4 & 20 & 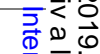 \\
\hline & & & & & & Total & 220 & 164 & 384 & 言市西 \\
\hline Tariku et al $2017(75)$ & Amhara, Dabat & Cross-sectional & Mothers with children aged & $5,227 / 5,227$ & University of & Uneducated & 286 & 268 & 554 & 을 \\
\hline & District & study * & less than 59 months & & Gondar & Primary & 57 & 86 & 143 & $\overline{\overline{\bar{n}}} \cong \overline{0}$ \\
\hline & & & & & & Secondary or above & 95 & 30 & 125 & $\overbrace{0}^{\infty} \frac{\pi}{2} \frac{0}{2}$ \\
\hline & & & & & & Total & 438 & 384 & 822 & 考它 \\
\hline Woldemichael et.al & Oromia, Tiyo & Cross-sectional & Mothers who have children & $386 / 373$ & Addis Ababa & Uneducated & 33 & 39 & 72 & $\overline{\bar{\tau}}$ \\
\hline $2016(73)$ & Woreda & study & less than one-year age & & University & Primary & 120 & 56 & 176 & $\frac{a}{c}$ \\
\hline & & & & & & Secondary or above & 98 & 27 & 125 & \\
\hline & & & & & & Total & 251 & 122 & 373 & $\bar{\tau}$ \\
\hline c. Household in & ome & & & & & & & & & \\
\hline Regassa, 2014(76) & SNNPR, Sidama & Cross-sectional & Mothers with infants aged & $1100 / 1094$ & Norwegian & Low & 670 & 166 & 836 & 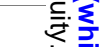 \\
\hline
\end{tabular}

Page 14 of 38 


\begin{tabular}{|c|c|c|c|c|c|c|c|c|c|c|}
\hline & \multirow[t]{3}{*}{ zone } & \multirow[t]{3}{*}{ study } & \multirow{3}{*}{$\begin{array}{l}\text { between } 0 \text { and } 6 \\
\text { months old }\end{array}$} & & \multirow{3}{*}{$\begin{array}{l}\text { Government (HU- } \\
\text { NORAD) }\end{array}$} & Medium & 185 & 47 & 232 & \\
\hline & & & & & & High & 22 & 5 & 27 & \\
\hline & & & & & & Total & 877 & 218 & 1095 & \\
\hline \multirow[t]{4}{*}{ Tariku et al 2017(75) } & \multirow{4}{*}{$\begin{array}{l}\text { Amhara, Dabat } \\
\text { District }\end{array}$} & \multirow{4}{*}{$\begin{array}{l}\text { Cross-sectional } \\
\text { study * }\end{array}$} & \multirow{4}{*}{$\begin{array}{l}\text { Mothers with children aged } \\
\text { less than } 59 \text { months }\end{array}$} & \multirow[t]{4}{*}{$5,227 / 5,227$} & \multirow{4}{*}{$\begin{array}{l}\text { University of } \\
\text { Gondar }\end{array}$} & Low & 103 & 204 & 307 & ర류 \\
\hline & & & & & & Medium & 172 & 97 & 269 & \\
\hline & & & & & & High & 163 & 83 & 246 & \\
\hline & & & & & & Total & 438 & 384 & 822 & \\
\hline \multirow[t]{4}{*}{ Ekubay et al 2018(62) } & \multirow{4}{*}{$\begin{array}{l}\text { Addis Ababa } \\
\text { Town }\end{array}$} & \multirow{4}{*}{$\begin{array}{l}\text { Cross-sectional } \\
\text { study }\end{array}$} & \multirow{4}{*}{$\begin{array}{l}\text { Mothers with infants } \\
\text { younger than or equal to six } \\
\text { months of age }\end{array}$} & \multirow[t]{4}{*}{$597 / 583$} & \multirow{4}{*}{$\begin{array}{l}\text { Addis Ababa } \\
\text { University }\end{array}$} & Low & 38 & 22 & 60 & $\begin{array}{l}\bar{\omega} \bar{\omega} \\
\overline{3}=\overline{\vec{\sigma}}\end{array}$ \\
\hline & & & & & & Medium & 241 & 185 & 426 & 。요 \\
\hline & & & & & & High & 50 & 28 & 78 & ڤั \\
\hline & & & & & & Total & 329 & 235 & 564 & \\
\hline \multirow{4}{*}{$\begin{array}{l}\text { Lakew et.al. } \\
2015(66)^{*}\end{array}$} & \multirow[t]{4}{*}{ National } & \multirow{4}{*}{$\begin{array}{l}\text { Cross-sectional } \\
\text { study }\end{array}$} & \multirow{4}{*}{$\begin{array}{l}\text { Mothers who had children } \\
\text { less than } 5 \text { years }\end{array}$} & \multirow[t]{4}{*}{$11,654 / 11,553$} & \multirow[t]{4}{*}{ Not mentioned } & Low & 2727 & 2498 & 5225 & $\bar{D} \bar{D}$ \\
\hline & & & & & & Medium & 1254 & 1125 & 2379 & \\
\hline & & & & & & High & 2212 & 1736 & 3948 & 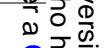 \\
\hline & & & & & & Total & 6193 & 5359 & 11552 & గై \\
\hline \multirow[t]{4}{*}{ John et al $2019(61)^{*}$} & \multirow[t]{4}{*}{ National } & \multirow{4}{*}{$\begin{array}{l}\text { Cross-sectional } \\
\text { study }\end{array}$} & \multirow{4}{*}{$\begin{array}{l}\text { Mothers with children less } \\
\text { than } 24 \text { months of age }\end{array}$} & \multirow[t]{4}{*}{$5,546 / 5,546$} & \multirow[t]{4}{*}{ No funding } & Low & 1399 & 467 & 1866 & 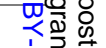 \\
\hline & & & & & & Medium & 648 & 219 & 867 & วై \\
\hline & & & & & & High & 1017 & 371 & 1388 & 记员文 \\
\hline & & & & & & Total & 3064 & 1057 & 4121 & 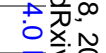 \\
\hline Berhe et.al 2013(64) & Tigray, Mekelle & Cross-sectional & Mothers of children aged 0 & $361 / 361$ & Addis Ababa & Low & 43 & 10 & 53 & 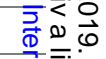 \\
\hline & town & study & to 24 months & & University & Medium & 90 & 22 & 112 & 总赵車 \\
\hline & & & & & & High & 128 & 33 & 161 & 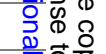 \\
\hline & & & & & & Total & 261 & 65 & 326 & 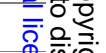 \\
\hline Seid 2014(77) & Amhara, Bahir & Cross-sectional & Mothers who delivered 12 & $819 / 819$ & University of & Low & 376 & 44 & 420 & 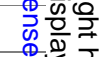 \\
\hline & Dar City & study & months before the study & & Gondar & Medium & 167 & 33 & 200 & $\neq \frac{0}{2}$ \\
\hline & & & began & & & High & 166 & 29 & 195 & $\frac{\pi}{0}$ \\
\hline & & & & & & Total & 709 & 106 & 815 & 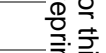 \\
\hline Tilahun et al & Amhara, Debre & Cross-sectional & mothers who had children & $416 / 409$ & University of & Low & 77 & 59 & 136 & 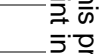 \\
\hline $2016(78)$ & Berhan town & study & less than six months of age & & Gondar & Medium & 113 & 71 & 184 & $\vec{\Phi}$ \\
\hline & & & & & & High & 66 & 23 & 89 & 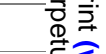 \\
\hline & & & & & & Total & 256 & 153 & 409 & 5 \\
\hline
\end{tabular}

Page 15 of 38 


\section{d. Marital status}

\begin{tabular}{|c|c|c|c|c|c|c|c|c|c|c|}
\hline \multirow[t]{3}{*}{ Tamiru et.al $2012(70)$} & \multirow{3}{*}{$\begin{array}{l}\text { Oromia, Jimma } \\
\text { Arjo Woreda }\end{array}$} & \multirow{3}{*}{$\begin{array}{l}\text { Cross-sectional } \\
\text { study }\end{array}$} & \multirow{3}{*}{$\begin{array}{l}\text { Mothers of index children } \\
\text { aged } 0 \text { to } 6 \text { months }\end{array}$} & \multirow[t]{3}{*}{$384 / 382$} & \multirow[t]{3}{*}{ Not mentioned } & Married & 231 & 137 & 368 & \\
\hline & & & & & & Other & 4 & 3 & 7 & \\
\hline & & & & & & Total & 235 & 140 & 375 & \\
\hline \multirow{3}{*}{$\begin{array}{l}\text { Beyene et.al } \\
2017(65)\end{array}$} & \multirow{3}{*}{$\begin{array}{l}\text { SNNPR, Dale } \\
\text { Woreda }\end{array}$} & \multirow{3}{*}{$\begin{array}{l}\text { Cross-sectional } \\
\text { study }\end{array}$} & \multirow{3}{*}{$\begin{array}{l}\text { Mothers of children under } \\
24 \text { months }\end{array}$} & \multirow[t]{3}{*}{$634 / 634$} & \multirow{3}{*}{$\begin{array}{l}\text { Addis Ababa } \\
\text { University }\end{array}$} & Married & 502 & 15 & 517 & \\
\hline & & & & & & Other & 96 & 5 & 101 & \\
\hline & & & & & & Total & 598 & 20 & 618 & \\
\hline \multirow{3}{*}{$\begin{array}{l}\text { Woldemichael et.al. } \\
2016(73)\end{array}$} & \multirow{3}{*}{$\begin{array}{l}\text { Oromia, Tiyo } \\
\text { Woreda }\end{array}$} & \multirow{3}{*}{$\begin{array}{l}\text { Cross-sectional } \\
\text { study }\end{array}$} & \multirow{3}{*}{$\begin{array}{l}\text { Mothers who have children } \\
\text { less than one-year age }\end{array}$} & \multirow[t]{3}{*}{$386 / 373$} & \multirow{3}{*}{$\begin{array}{l}\text { Addis Ababa } \\
\text { University }\end{array}$} & Married & 244 & 117 & 361 & \\
\hline & & & & & & Other & 7 & 5 & 12 & \\
\hline & & & & & & Total & 251 & 122 & 373 & 焉实员 \\
\hline \multirow[t]{3}{*}{ Berhe et.al 2013(64) } & \multirow{3}{*}{$\begin{array}{l}\text { Tigray, Mekelle } \\
\text { town }\end{array}$} & \multirow{3}{*}{$\begin{array}{l}\text { Cross-sectional } \\
\text { study }\end{array}$} & \multirow{3}{*}{$\begin{array}{l}\text { Mothers of children aged } 0 \\
\text { to } 24 \text { months }\end{array}$} & \multirow[t]{3}{*}{$361 / 361$} & \multirow{3}{*}{$\begin{array}{l}\text { Addis Ababa } \\
\text { University }\end{array}$} & Married & 256 & 71 & 327 & 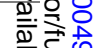 \\
\hline & & & & & & Other & 22 & 8 & 30 & \\
\hline & & & & & & Total & 278 & 79 & 357 & 등 \\
\hline \multirow[t]{3}{*}{ Derso et.al $2017(60)^{*}$} & \multirow{3}{*}{$\begin{array}{l}\text { Amhara, Dabat } \\
\text { district }\end{array}$} & \multirow{3}{*}{$\begin{array}{l}\text { Cross-sectional } \\
\text { study }\end{array}$} & \multirow{3}{*}{$\begin{array}{l}\text { Mothers with children } \\
\text { under five years of age }\end{array}$} & \multirow[t]{3}{*}{$6,761 / 6,761$} & PEPFAR-CDC & Married & 2524 & 3359 & 5883 & $\frac{\mathbb{Q}}{\infty}$ \\
\hline & & & & & & Other & 445 & 433 & 878 & \\
\hline & & & & & & Total & 2969 & 3792 & 6761 & \\
\hline John et al $2019(61)^{*}$ & National & Cross-sectional & Mothers with children less & $5,546 / 5,546$ & No funding & Married & 2898 & 981 & 3879 & 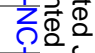 \\
\hline & & study & than 24 months of age & & & Other & 166 & 76 & 242 & 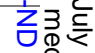 \\
\hline & & & & & & Total & 3064 & 1057 & 4121 & 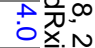 \\
\hline Liben et.al 2016(67) & Afar, Dubti & Cross-sectional & Mothers of infants aged less & $346 / 333$ & Samara University & Married & 124 & 158 & 282 & \\
\hline & town & study & than 6 months & & & Other & 27 & 72 & 99 & $\bar{D}_{0}$ \\
\hline & & & & & & Total & 151 & 230 & 381 & 言哥市 \\
\hline Setegn et.al 2011(68) & Oromia, Goba & Cross sectional & Mothers with child ren $k 12$ & $668 / 608$ & Jimma University & Married & 300 & 270 & 570 & \\
\hline & district & study & months & & & Other & 14 & 15 & 29 & \\
\hline & & & & & & Total & 314 & 285 & 599 & 흠 \\
\hline Tewabe $2016(79)$ & Amhara, Motta & Cross-sectional & Mothers with infant less & $423 / 405$ & Addis Ababa & Married & 280 & 67 & 347 & D \\
\hline & town & study & than six-month-old & & University & Other & 38 & 19 & 57 & \\
\hline & & & & & & Total & 318 & 86 & 404 & ס \\
\hline Ekubay et al 2018(62) & Addis Ababa & Cross-sectional & mothers with infants $\leq$ six & $597 / 583$ & Addis Ababa & Married & 295 & 213 & 508 & 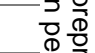 \\
\hline & Town & study & months of age & & University & Other & 34 & 22 & 56 & 总 \\
\hline & & & & & & Total & 329 & 235 & 564 & $\underline{\underline{z}}$ \\
\hline
\end{tabular}

Page 16 of 38

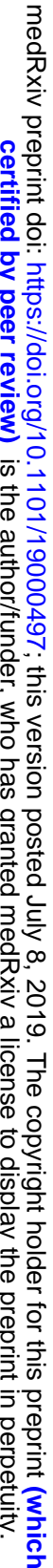




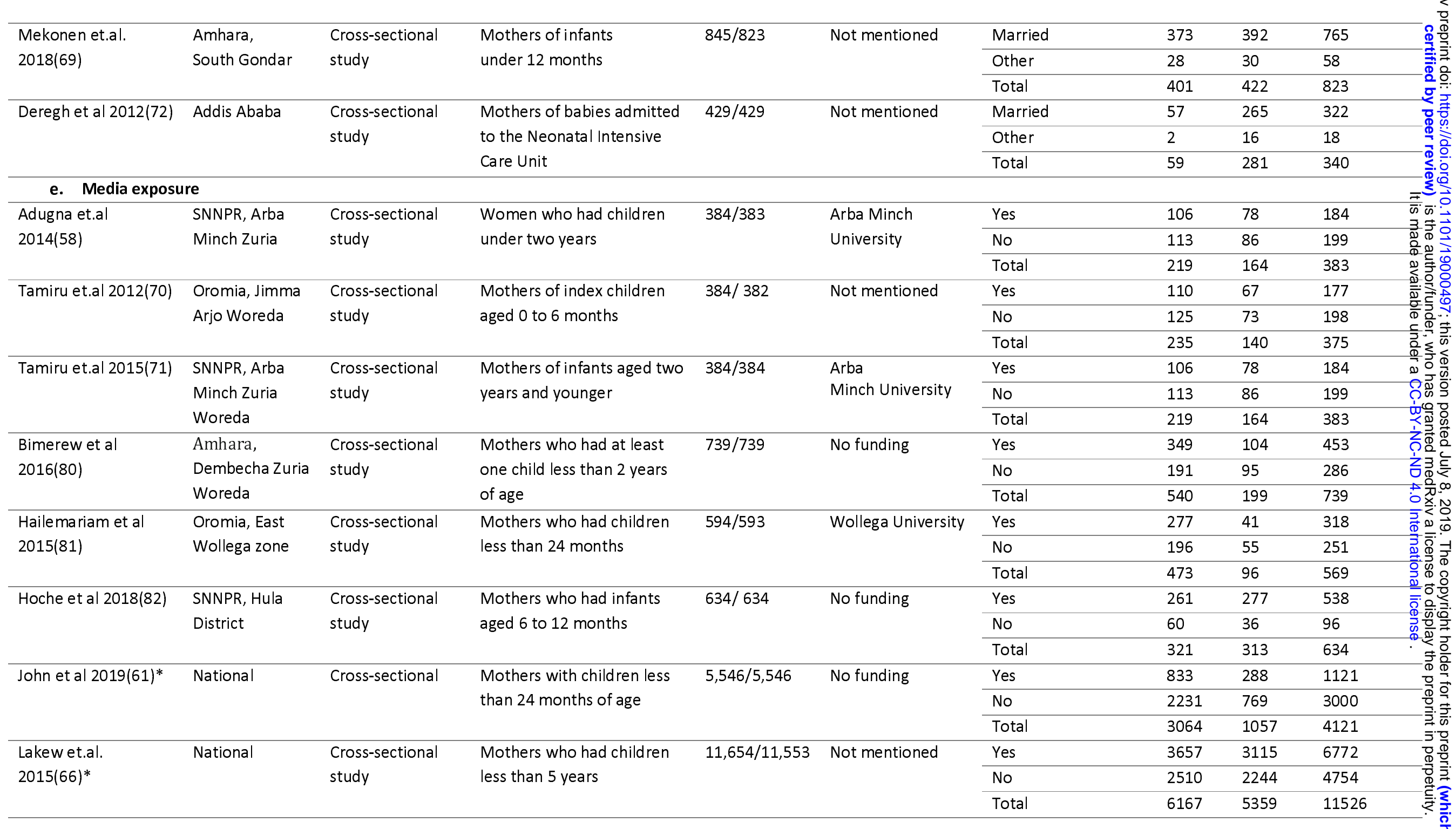

Page 17 of 38 


\begin{tabular}{|c|c|c|c|c|c|c|c|c|c|c|}
\hline Beyene et.al & SNNPR, Dale & Cross-sectional & Mothers of children under & $634 / 634$ & Addis Ababa & Yes & 304 & 80 & 384 & \\
\hline \multirow[t]{2}{*}{$2017(65)$} & \multirow[t]{2}{*}{ Woreda } & \multirow[t]{2}{*}{ study } & \multirow[t]{2}{*}{24 months } & & \multirow[t]{2}{*}{ University } & No & 213 & 21 & 234 & \\
\hline & & & & & & Total & 517 & 101 & 618 & \\
\hline \multirow[t]{3}{*}{ Ekubay et al 2018(62) } & \multirow{3}{*}{$\begin{array}{l}\text { Addis Ababa } \\
\text { Town }\end{array}$} & \multirow{3}{*}{$\begin{array}{l}\text { Cross-sectional } \\
\text { study }\end{array}$} & \multirow{3}{*}{$\begin{array}{l}\text { mothers with infants } \leq \text { six } \\
\text { months of age }\end{array}$} & \multirow[t]{3}{*}{$597 / 583$} & \multirow{3}{*}{$\begin{array}{l}\text { Addis Ababa } \\
\text { University }\end{array}$} & Yes & 250 & 183 & 433 & \\
\hline & & & & & & No & 79 & 52 & 131 & \\
\hline & & & & & & Total & 329 & 235 & 564 & \\
\hline \multirow{3}{*}{$\begin{array}{l}\text { Mekonen et.al. } \\
\text { 2018(69) }\end{array}$} & \multirow{4}{*}{$\begin{array}{l}\text { Amhara, } \\
\text { South Gondar }\end{array}$} & \multirow{4}{*}{$\begin{array}{l}\text { Cross-sectional } \\
\text { study }\end{array}$} & \multirow{4}{*}{$\begin{array}{l}\text { Mothers of infants } \\
\text { under } 12 \text { months }\end{array}$} & \multirow[t]{4}{*}{$845 / 823$} & \multirow[t]{4}{*}{ Not mentioned } & Yes & 312 & 259 & 571 & $=$ है \\
\hline & & & & & & No & 89 & 163 & 252 & $\begin{array}{l}\bar{\omega} \\
\overline{3}\end{array}$ \\
\hline & & & & & & Total & 401 & 422 & 823 & \\
\hline \multicolumn{5}{|l|}{ f. Parity } & & & & & & \\
\hline Beyene et.al & SNNPR, Dale & Cross-sectional & Mothers of children under & \multirow[t]{3}{*}{$634 / 634$} & \multirow{3}{*}{ University } & Primiparous & 176 & 57 & 233 & \\
\hline \multirow[t]{2}{*}{$2017(65)$} & \multirow[t]{2}{*}{ Woreda } & \multirow[t]{2}{*}{ study } & \multirow[t]{2}{*}{24 months } & & & Multiparous & 341 & 44 & 385 & \\
\hline & & & & & & Total & 517 & 101 & 618 & 동 \\
\hline \multirow[t]{3}{*}{ Liben et.al 2016(67) } & \multirow{3}{*}{$\begin{array}{l}\text { Afar, Dubti } \\
\text { town }\end{array}$} & \multirow{3}{*}{$\begin{array}{l}\text { Cross-sectional } \\
\text { study }\end{array}$} & \multirow{3}{*}{$\begin{array}{l}\text { Mothers of infants aged less } \\
\text { than } 6 \text { months }\end{array}$} & $346 / 333$ & Samara University & Primiparous & 29 & 65 & 94 & D일 \\
\hline & & & & & & Multiparous & 122 & 165 & 287 & \\
\hline & & & & & & Total & 151 & 230 & 381 & \\
\hline Ekubay et al 2018(62) & Addis Ababa & Cross-sectional & mothers with infants $\leq$ six & $597 / 583$ & Addis Ababa & Primiparous & 199 & 160 & 359 & לฺุ \\
\hline & Town & study & months of age & & University & Multiparous & 130 & 75 & 205 & \\
\hline & & & & & & Total & 329 & 235 & 564 & 辛要 \\
\hline Setegn et.al 2011(68) & Oromia, Goba & Cross sectional & Mothers with children $(<12$ & $668 / 608$ & Jimma University & Primiparous & 83 & 63 & 146 & \\
\hline & district & study & months & & & Multiparous & 226 & 223 & 449 & \\
\hline & & & & & & Total & 309 & 286 & 595 & \\
\hline Mekonen et.al. & Amhara, & Cross-sectional & Mothers of infants & $845 / 823$ & Not mentioned & Primiparous & 163 & 233 & 396 & $\overline{\overline{\bar{n}}} \cong$ \\
\hline $2018(69)$ & South Gondar & study & Under 12 months & & & Multiparous & 238 & 189 & 427 & \\
\hline & & & & & & Total & 401 & 422 & 823 & \\
\hline Woldemichael et.al & Oromia, Tiyo & Cross-sectional & Mothers who have children & $386 / 373$ & Addis Ababa & Primiparous & 88 & 43 & 131 & 元 \\
\hline $2016(73)$ & Woreda & study & less than one-year age & & University & Multiparous & 163 & 79 & 242 & $\frac{\pi}{0}$ \\
\hline & & & & & & Total & 251 & 122 & 373 & 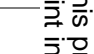 \\
\hline Hoche et al 2018(82) & SNNPR, Hula & Cross-sectional & Mothers who had infants & $634 / 634$ & No funding & Primiparous & 183 & 228 & 411 & $\bar{\pi}$ \\
\hline & District & study & aged 6 to 12 months & & & Multiparous & 138 & 85 & 223 & \\
\hline & & & & & & Total & 321 & 313 & 634 & 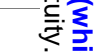 \\
\hline
\end{tabular}

Page 18 of 38 


\begin{tabular}{|c|c|c|c|c|c|c|c|c|}
\hline \multirow[t]{3}{*}{ Gultie et.al 2016(59) } & \multirow{3}{*}{$\begin{array}{l}\text { Amhara, Debre } \\
\text { Berhan town }\end{array}$} & \multirow{3}{*}{$\begin{array}{l}\text { Cross-sectional } \\
\text { study }\end{array}$} & Mothers having children & \multirow[t]{3}{*}{$548 / 548$} & \multirow[t]{3}{*}{ Not mentioned } & Primiparous & 158 & 34 \\
\hline & & & Aged less than 23 months & & & Multiparous & 286 & 70 \\
\hline & & & old & & & Total & 444 & 104 \\
\hline \multirow[t]{3}{*}{ Deregh et al 2012(72) } & \multirow[t]{3}{*}{ Addis Ababa } & \multirow{3}{*}{$\begin{array}{l}\text { Cross-sectional } \\
\text { study }\end{array}$} & Mothers of babies admitted & \multirow[t]{3}{*}{$429 / 429$} & \multirow[t]{3}{*}{ Not mentioned } & Primiparous & 29 & 155 \\
\hline & & & to the Neonatal Intensive & & & Multiparous & 30 & 126 \\
\hline & & & Care Unit & & & Total & 59 & 281 \\
\hline
\end{tabular}

\section{*= Used Ethiopian Demographic Health Survey (EDHS) data; PEPFAR = The President's Emergency Plan for AIDS Relief; CDC = the Centers for}

Disease Control and Prevention (CDC); NORAD = Norwegian Agency for Development Cooperation; SNNPR = Southern Nations, Nationalities, and Peoples' Region 


\section{Predicting factors}

\section{Maternal educational status}

Seventeen studies(58-74) involving 31,066 mothers reported the association between maternal educational status and TIBF (Table 1a). Of which, four studies were conducted in Amhara region, three in Oromia region, three studies in Southern Nations, Nationalities and Peoples Region (SNNPR), and five studies in other regions (Addis Ababa, Afar, and Tigray). Two studies used nationally representative data. The odds of TIBF among mothers who attained primary education was $23 \%$ significantly higher than uneducated mothers (OR $=$ $1.23 ; \mathrm{p}=0.02 ; 95 \% \mathrm{Cl}=1.04-1.47 ; \mathrm{I}^{2}=80.89 \%$ ) (Figure 1). The result of meta-regression analysis showed that region, residence, sample size and publication year were not the source of between-study heterogeneity. The odds of TIBF among mothers with secondary education or above was $82 \%$ significantly higher than uneducated mothers $(O R=1.82 ; \mathrm{p}<$ $0.001 ; 95 \% \mathrm{Cl}=1.35-2.45 ;\left.\right|^{2}=84.96 \%$ ) (Supplementary Figure S1). The meta-regression analysis showed that $78.68 \%$ of the between-study heterogeneity explained by variation in region and publication year. Region had significant moderation effect $(Q M=22.72, d f=6, p$ $<0.01$ ). Additionally, the odds of TIBF among mothers with secondary education or above was $43 \%$ significantly higher than mothers who attained primary education (OR $=1.43 ; p=$ $0.002 ; 95 \% \mathrm{Cl}=1.15-1.79, \mathrm{I}^{2}=73.03 \%$ ) (Supplementary Figure S2). In all three comparisons, there was no significant publication bias (Supplementary Figures S3, S4, S5) and the evidence on the effect of maternal educational status on TIBF did not markedly change over time (Supplementary Figures S6, S7, S8). Overall, we observed a direct dose-response relationship between maternal educational status and TIBF. 


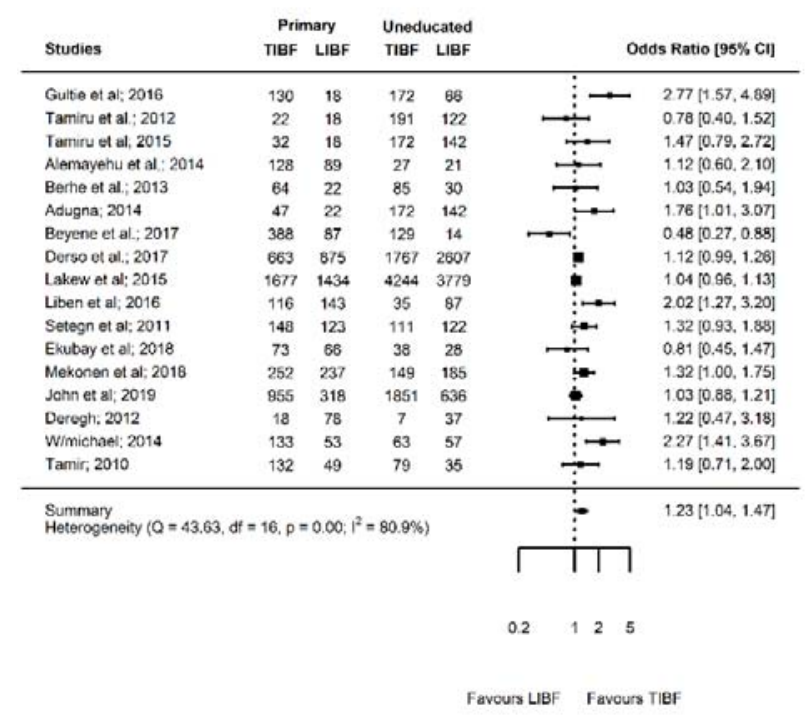

Figure 1: Forest plot showing the results of 17 studies examining the association between maternal educational status (primary education versus uneducated) and timely initiation of breast feeding.

\section{Paternal educational status}

Four studies $(61,71,73,75)$ with 11,491 mothers reported the association between paternal educational status and TIBF (Table 1b). These studies were conducted in Amhara, Oromia, and SNNPR. One study used nationally representative data. The odds of TIBF in mothers who had a spouse with primary education was $17 \%$ higher than mothers who had an uneducated spouse, although the difference was not statistically significant $(O R=1.17 ; p=$ $0.56 ; 95 \% \mathrm{Cl}=0.69-2.01, \mathrm{I}^{2}=89 \%$ ) (Supplementary Figure S9). The meta-regression analysis revealed that $12.68 \%$ of between-study heterogeneity due to the difference in residence and publication year of studies, however, moderation effect was not observed. Mothers who had a spouse with secondary education or above were 2.72 times significantly higher chance of TIBF compared with mothers who had an uneducated spouse (OR $=2.72 ; \mathrm{p}=$ $0.001,95 \% \mathrm{Cl}=1.49-4.97 \mathrm{I}^{2}=62.50 \%$ ) (Figure 2). This result was obtained after removing one outlier study(61) using Jackknife analysis. Moreover, the odds of TIBF in mothers who had a spouse with secondary education or above was $66 \%$ higher than mothers who had had a spouse with primary education although this difference was not statistically significant (OR $=1.66 ; p=0.21 ; 95 \% \mathrm{Cl}=0.75-3.66, \mathrm{I}^{2}=90.44 \%$ ) (Supplementary Figure S10). The meta-regression analysis revealed that $12.68 \%$ of between-study heterogeneity can be 
explained by the difference in the residence but had no moderation effect. In all comparisons, there was no significant publication bias (Supplementary Figures S11, S12, S13). The evidence on the effect of paternal educational status on TIBF was mixed with dramatic decrement (Supplementary Figures S14 and S15) and stability (Supplementary Figure S16) over time. Similar to maternal educational status, there was a clear indication of the dose-response relationship between paternal educational status and TIBF, although the association was not consistent at different grade level.

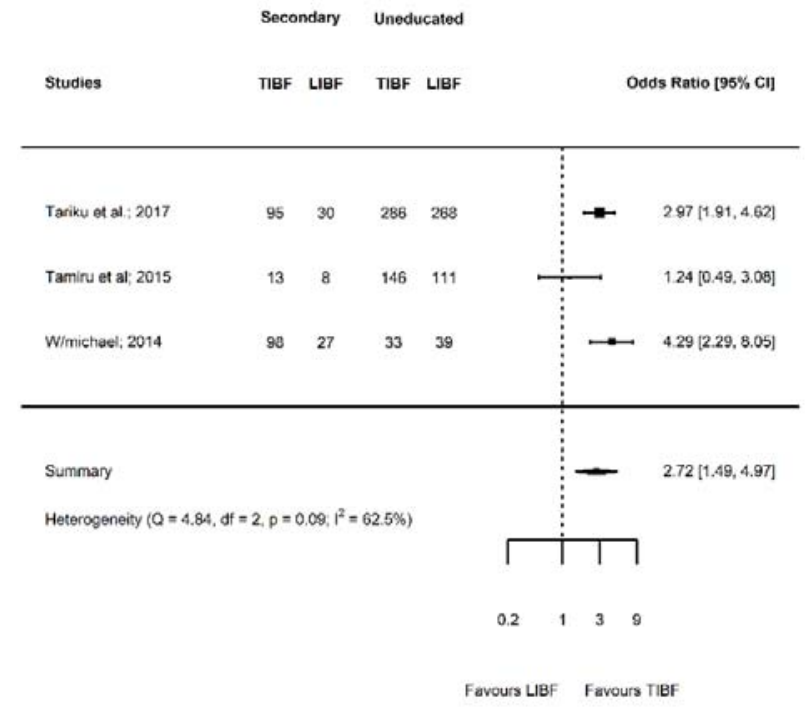

Figure 2: Forest plot showing the results of four studies examining the association between paternal educational status (Secondary or above versus uneducated) and timely initiation of breast feeding.

\section{Household income}

Eight studies $(61,62,64,66,75-78)$ including 21,265 mothers reported the association between income and TIBF (Table 1c). Three studies conducted in Amhara region and the remaining three studies were from Addis Ababa, Tigray, and SNNPR. Two studies used nationally representative data. The odds of TIBF among mothers who had medium income was $10 \%$ higher than mothers who had low income $(O R=1.10 ; p=0.64 ; 95 \% \mathrm{Cl}=0.75$ $1.60 ; I^{2}=92.44 \%$ ) (Supplementary Figure S17). The meta-regression analysis showed that the heterogeneity fully accounted for variation in region and residence, and these factors had a moderation effect as well $(\mathrm{QM}=50.72, \mathrm{df}=5, \mathrm{p}<0.001)$. The odds of TIBF among mothers who had high income was $28 \%$ higher than mothers who had low income (OR = 
1.28; $\mathrm{p}=0.25 ; 95 \% \mathrm{Cl}=0.84-1.96 ; \mathrm{I}^{2}=93.87 \%$ ) (Supplementary Figure S18). The metaregression analysis showed that $91.50 \%$ of the heterogeneity accounted for the variation in the region, residence, and sample size. These factors had also moderation effect ( $Q M=$ 32.24, $d f=6, p<0.01$ ). The odds of TIBF among mothers who had high income was $16 \%$ significantly higher than mothers who had medium income $(O R=1.16 ; p=0.002 ; 95 \% \mathrm{Cl}=$ $1.05-1.27 ; I^{2}=0.00 \%$ ) (Figure 3). This was the estimate after excluding one outlier study(61) using Jackknife sensitivity analysis. There was no significant publication bias in all comparisons (Supplementary Figures S19, S20, S21). The evidence on the effect of income on TIBF was slightly increased over time (Supplementary Figures S22, S23, S24). Overall, there was a reverse dose-response relationship between income and TIBF, although the association was not consistently significant.

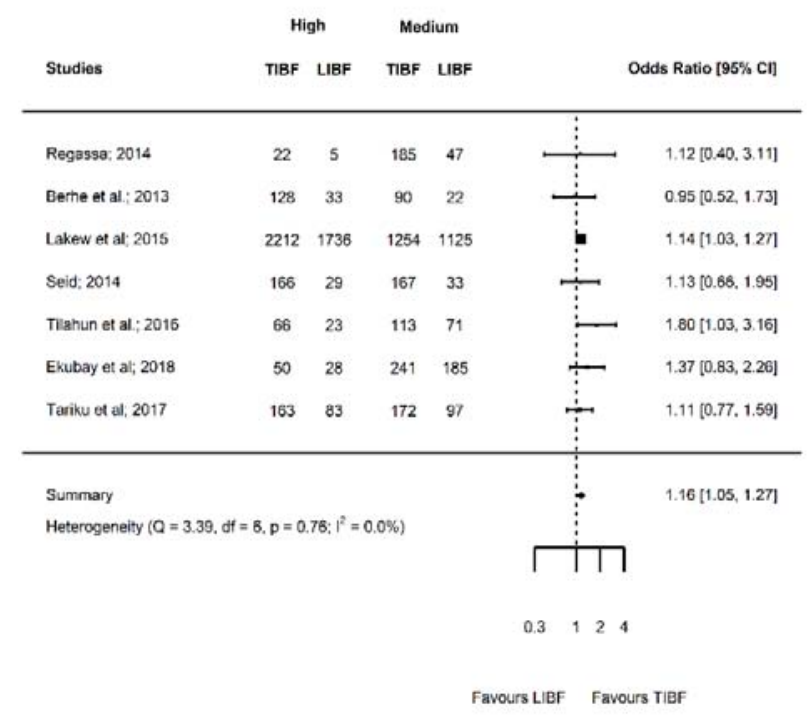

Figure 3: Forest plot showing the results of eight studies examining the association between household income (high versus medium) and timely initiation of breast feeding.

\section{Marital status}

Twelve studies(60-62, 64, 65, 67-70, 72, 73, 79) involving 17,259 mothers reported the association between marital status and TIBF (Table $1 \mathrm{~d}$ ). Three studies were conducted in Amhara region, three in Oromia region and the rest were conducted in Addis Ababa, Afar, and Tigray while one study was nationally conducted. One outlier study(60) was excluded after Jackknife sensitivity analysis. The odds of TIBF among married mothers was $39 \%$ significantly higher than unmarried mothers $\left(O R=1.39 ; p=0.001 ; 95 \% \mathrm{Cl}=1.14-1.69 ; I^{2}=\right.$ 
9.17\%) (Figure 4). There was no significant publication bias (Supplementary Figure S25). The evidence on the effect of marital status on TIBF was slightly increased over time (Supplementary Figure S26).

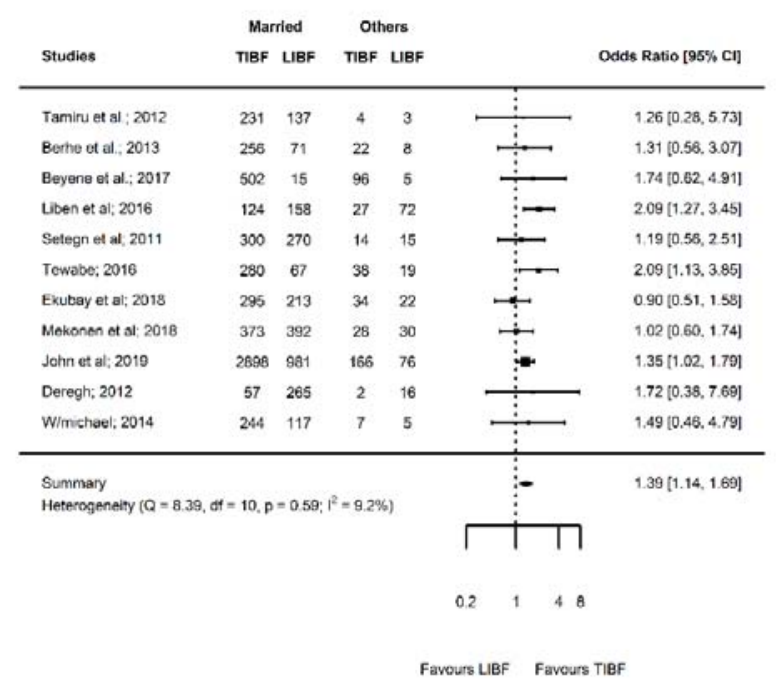

Figure 4: Forest plot showing the results of 11 studies examining the association between marital status (married versus others) and timely initiation of breast feeding.

\section{Media exposure}

Eleven studies $(58,61,62,65,66,69-71,80-82)$ with 22,315 mothers reported the association between media exposure and TIBF (Table 1e). Four studies were conducted in SNNPR, two studies in Amhara region, two studies in Oromia region and one study in Addis Ababa. Two studies used nationally representative data. The odds of TIBF among mothers who were exposed to media was $5 \%$ higher than mothers who were not exposed media, although the difference was not statistically significant $\left(\mathrm{OR}=1.05 ; \mathrm{p}=0.74 ; 95 \% \mathrm{Cl}=0.79-1.40 ; \mathrm{I}^{2}=\right.$ 92.15\%) (Figure 5). There was no significant publication bias (Supplementary Figure S27). The evidence on the effect of media exposure on TIBF over time was stable (Supplementary Figure S28). The meta-regression analysis result showed that $62.61 \%$ of the between-study heterogeneity accounted for the variation in region and publication year, however, none of these factors had moderation effect ( $Q M=16.44, d f=5, p=0.05)$. 


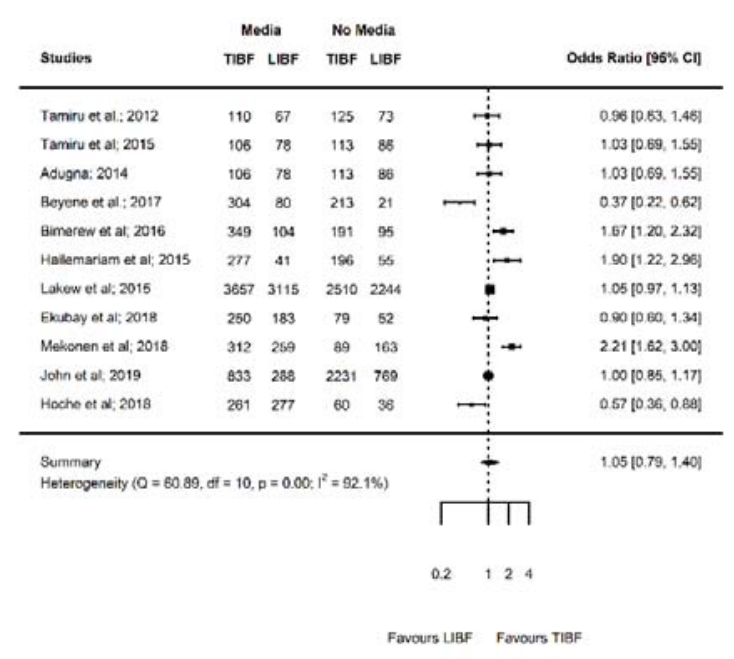

Figure 5: Forest plot showing the results of 11 studies examining the association between media exposure (yes versus no) and timely initiation of breast feeding.

\section{Parity}

Nine studies $(59,62,65,67-69,72,73,82)$ with 4,993 mothers reported the association between parity and TIBF (Table 1f). Of these, two studies were conducted in Addis Ababa, two in Amhara, two in Oromia, two in SNNPR and one in Afar region. The odds of TIBF among multiparous mothers was $39 \%$ significantly higher than primiparous mothers $(O R=1.39 ; p=$ $0.01 ; 95 \% \mathrm{Cl}=1.07-1.81 ; I^{2}=74.43 \%$ ) (Figure 6). There was no significant publication bias (Supplementary Figure S29). The evidence on the effect of parity on TIBF was steadily increased over time (Supplementary Figure S30). 
medRxiv preprint doi: https://doi.org/10.1101/19000497; this version posted July 8, 2019. The copyright holder for this preprint (which was not certified by peer review) is the author/funder, who has granted medRxiv a license to display the preprint in perpetuity. It is made available under a CC-BY-NC-ND 4.0 International license.

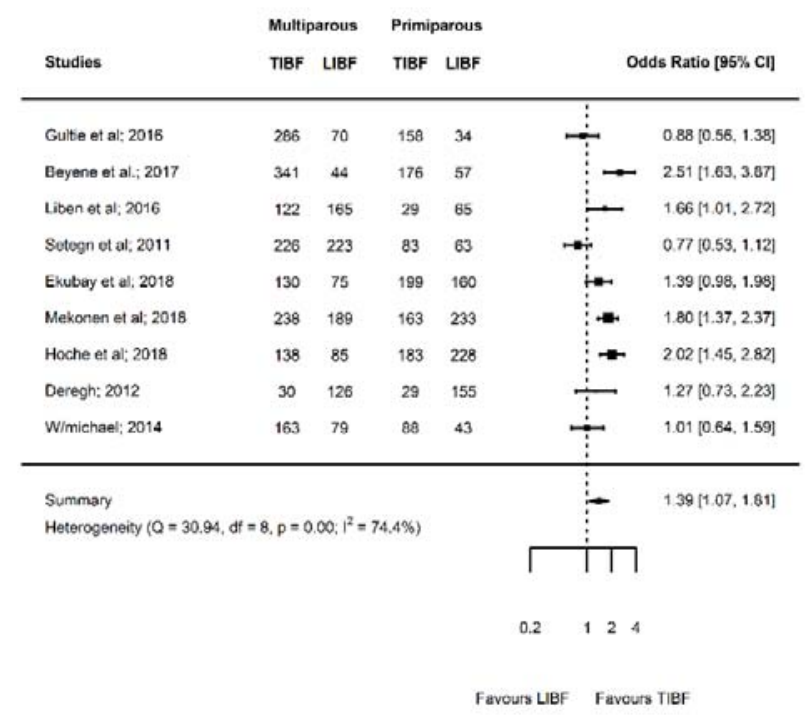

Figure 5: Forest plot showing the results of nine studies examining the association between parity (multiparous versus primiparous) and timely initiation of breast feeding. 
medRxiv preprint doi: https://doi.org/10.1101/19000497; this version posted July 8, 2019. The copyright holder for this preprint (which was not certified by peer review) is the author/funder, who has granted medRxiv a license to display the preprint in perpetuity.

It is made available under a CC-BY-NC-ND 4.0 International license .

\section{Discussion}

This meta-analysis unraveled the association between timely initiation of breast feeding (TIBF) and maternal educational status, paternal educational status, household income, marital status, media exposure, and parity. The key findings were TIBF significantly associated with maternal educational status, paternal educational status, household income, marital status, and parity. We further observed a clear dose-response relationship of TIBF with educational status and household income.

In this study, the odds of TIBF among mothers with primary education was significantly higher than uneducated mothers. The association was even stronger when we compare mothers with secondary education or above with uneducated mothers and mothers who attained primary education, which clearly shows a direct dose-relationship. We observed a similar association between paternal educational status and TIBF. This finding was in line with our hypothesis and studies in India, Nepal, and Timor-Leste. $(83,84)$ It is likely that education plays an important role in changing the beliefs and attitudes of mothers toward breast feeding, increasing confidence, increase spousal support, maximize ANC follow-up, increase maternal decision making and a higher likelihood of delivering at health institution. In addition, highly educated mothers and fathers could have high expectation, pose questions and request to be properly counselled. On the other hand, other studies $(31,32,85)$ reported the absence of a significant association between education status and TIBF. Another study(86) revealed that high educational status found to be associated with delayed initiation of breast feeding. This inconsistency might be attributed to the difference in the study population, sample size and socioeconomic status. The pooled results of our meta-analysis and others (India, Nepal, and Timor-Leste) are based on nationally available studies or data. $(83,84)$ In addition, delayed initiation of breast feeding in highly educated mothers may be due to the use of private maternities and the larger rate of caesarean section.(87)

In agreement with previous studies $(31,87)$, we also showed mothers who had high income are likely to pratice TIBF compared with mothers with low income. This might be due to wealthier mothers are more likely to follow the recommended level of antenatal care visits, deliver at health institution and receive adequate postnatal care which can help them to timely initiate breast feeding, whereas poor mothers are deprived of those economic advantages.(88-90) In our previous meta-analyses(30,40, 41), we affirmed that antenatal 
medRxiv preprint doi: https://doi.org/10.1101/19000497; this version posted July 8, 2019. The copyright holder for this preprint (which was not certified by peer review) is the author/funder, who has granted medRxiv a license to display the preprint in perpetuity. It is made available under a CC-BY-NC-ND 4.0 International license .

care, health institution delivery, and postnatal care significantly associated with optimal breast feeding. In contrast, evidence from Timor-Leste demographic and health survey showed that income was not associated with TIBF.(85)

Moreover, our meta-analysis also showed that currently married mothers timely initiate breast feeding compared with currently unmarried (i.e. single, widowed, divorced) mothers. This finding was supported by previous longitudinal family-based study.(91) This may result from several reasons. Married mothers could have higher incomes and educational levels(92), as we showed socioeconomic advantage increased likelihood of TIBF. In addition, previous study has shown that married mothers are more satisfied, committed to spousal relationships, get shared spousal support and reported less conflict, and therefore, married mothers can have high emotional responsibility to keep the health of the newborn and more likely to engage in positive parenting behaviours.(93) In contrast, a systematic review by Esteves and colleagues reported the absence of association between marital status and initiation of breast feeding in 10 out of 12 studies.(87) This discrepancy might be due to difference in cultural significance of marriage, as a result, the rate of marriage is low or high.

In this study, we further found a significant positive association between multiparity and TIBF. This finding supported our hypothesis and is similar to the evidence in Middle East countries.(32) This may be due to the knowledge advantage that experience give to multiparous women compared with the inexperienced primiparous women. There is evidence that multiparity is associated with better breastfeeding related knowledge.(94) In addition, the increased knowledge and experience may change maternal attitude and behaviour towards breast feeding. On the contrary, a systematic review reported the absence of association between parity and initiation of breast feeding.(87)

In this meta-analysis, we depicted the relevant effect of maternal and paternal education, income, marital status, and parity ob breast feeding initiation. Therefore, health professionals should give special emphasis on uneducated or less educated parents with alternative supportive and educational interventions, such as prenatal education, counseling, and peer education.(83) School education is also required for young girls to increase breast feeding awareness and to prepare for motherhood.(83) Maternal education plays an important role in infant feeding behavioral change and to maintain the mother and 
medRxiv preprint doi: https://doi.org/10.1101/19000497; this version posted July 8, 2019. The copyright holder for this preprint (which was not certified by peer review) is the author/funder, who has granted medRxiv a license to display the preprint in perpetuity. It is made available under a CC-BY-NC-ND 4.0 International license .

newborn health. Even though there has been huge improvements in womens education in Ethiopia, it has long been neglected and remains much lower than men. In addition, interventions targeting fathers at antenatal and postnatal periods may increase breast feeding practices, and should be incorporated in breast feeding programs.(95) Improving the economic power of women would also increase TIBF practice. Furthermore, health professionals should give attention to single and primiparous mothers before, during and after birth. The training of health community agents and structural changes in health services might be relevant to increase TIBF practice.(87)

This meta-analysis showed the strength of association and provided national evidence as previous national, regional, or international studies lacked to report the pooled effect of various relevant factors affecting TIBF. Thus, future researchers in low- and middleincome countries can focus on meta-analysis instead of narrative review. To minimize the possibility of missing relevant studies, we have used a combination of electronic databases search and manual search of cross-references, grey literature, and table of contents of relevant journals. Furthermore, this meta-analysis was conducted based on a published protocol to minimize methodological biases.

We admitted that this meta-analysis has also limitations, which should be taken in to account during the translation of results. All the studies included in this meta-analysis were cross-sectional study, which hinders inference on a cause-effect relationship. The risk of measurement error and recall bias should also be acknowledged. Interestingly, almost all included studies were conducted in mothers with a newborn less than 23 months. In relation to this, the maternal recall is found to be a valid and reliable estimate of breast feeding initiation and duration when the data is collected within three years of breast feeding history.(96) Social desirability bias could also be evident given that self-reported breast feeding experience, educational status and income was used. In addition, the household income classifications are not standardized and did not account for indices such as inflation, which changes over time. Another problem was the confounding effect that can not be excluded given that the reported effect size in this meta-analysis was not adjusted to covariates, such as breast feeding counseling during ANC and PNC follow-up. Studies were lacking in some regions for some variables, which may limit generalizability of our findings. Despite this fact, at least one study used nationally representative data per each variable. In 
medRxiv preprint doi: https://doi.org/10.1101/19000497; this version posted July 8, 2019. The copyright holder for this preprint (which was not certified by peer review) is the author/funder, who has granted medRxiv a license to display the preprint in perpetuity. It is made available under a CC-BY-NC-ND 4.0 International license .

addition, only four studies investigated the association between paternal educational status and TIBF, which may limits the statistical power of our meta-analysis. This meta-analysis only covers studies in Ethiopia; therefore, a comparative meta-analysis from other LMCs and developed countries is required. Moreover, statistical heterogeneity was observed in some of the analysis. Even though residual heterogeneity is still evident, we managed this problem by conducting meta-regression analysis and by removing outlier studies using the Jackknife method. In addition, clinical heterogeneity was minimized by including only studies conducted on healthy mothers and newborns. Methodological heterogeneity was minimized by including studies that reported TIBF based on WHO definition, selected study subjects using a random sampling method, and similar study design and data collection methods. The dose-response relationship of multiparity and TIBF was not investigated due to the huge difference between included studies in the categorization of multiparity. A further limitation of this study was maternal and paternal education represents the formal education gained through schooling and it may not reflect the health literacy of the mothers and fathers. Finally, the results cannot be extrapolated to mothers and newborns with HIV/AIDS or other medical illness.

Measuring breast feeding is challenging, and the use of standardized questions may be interpreted differently according to sociocultural contexts and differing probing techiniques by interviewers.(97) In developing countries, breast feeding coverage is relatively good but we noted studies focused only on factors affecting breast feeding. To brideg this gap, future studies in Ethiopia should focus on investigating the effect of breast feeding on maternal and newborn health outcomes, such as cardiometabolic diseases, neuropsychological diseases, and psychiatric disorders. All included studies in the current review performed the traditional bivariate and multiple logistic regression. As a result, studies on associated factors should deeply consider the interconnection between predictors and apply detailed statistical analysis methods, such as mediation and moderation analysis. For example, it is suggested that geographical, socioeconomic, individual, and health-specific levels factors lower TIBF practice by influencing prelacteal feeding and discarding colostrum, accessibility of information and media, perception, support and milk sufficiency, and involvement of mothers in decision making.(34) Moreover, future research should examine the possible causations between predicting factors and TIBF 
practices in Ethiopia by implementing longitudinal research designs and large cohort-based studies, which can therefore, produce more accurate and insightful results. Overall, we observed a substantial inconsistency regarding predicting factors across nations; therefore, context-specific meta-analysis is required to strengthen inferences.

To concluded, proximal and distal factors significantly predicting TIBF practice in Ethiopia, which needs integrated intervention by health professionals and healthcare policymakers. Health education, counselling and peer education targeting parents at antenatal and postnatal periods are needed. It is also relevant to improve the economic power of women and promote gender equality.

\section{Acknowledgment}

We would like to Sjoukje van der Werf (librarian at the University Medical Center Groningen, the Netherlands) for her expert advice on search strategies and Balewgizie Sileshi (University of Groningen, the Netherlands) for his help to screen titles and abstracts.

\section{Data sharing statement}

All data generated or analysed in this study are included in the article and its supplementary files.

\section{Contributors}

TD conceived and designed the study, analyzed the data and interpreted the results. TD and SM extracted the data and wrote the first draft of the manuscript. All authors involved in writing, revising and providing intellectual comments, and approved the final manuscript.

\section{Funding}

This research received no specific grant from any funding agency in the public, commercial or not-for-profit sectors.

\section{Competing interests}

None declared. 
medRxiv preprint doi: https://doi.org/10.1101/19000497; this version posted July 8, 2019. The copyright holder for this preprint (which was not certified by peer review) is the author/funder, who has granted medRxiv a license to display the preprint in perpetuity.

It is made available under a CC-BY-NC-ND 4.0 International license.

1. . Breastfeeding: nutrition. http://www.unicef.org/nutrition/index 24824.html. August/05 2016

2. WHO Secretariat. Early Initiation of Breastfeeding: the Key to Survival and Beyond 2010

3. UNICEF W. Capture the Moment - Early initiation of breastfeeding: The best start for every newborn. 2018

4. Bernard JY, Armand M, Garcia C, et al. The association between linoleic acid levels in colostrum and child cognition at 2 and $3 y$ in the EDEN cohort. Pediatr Res 2015;77(6):829

5. Khan J, Vesel L, Bahl R, Martines JC. Timing of breastfeeding initiation and exclusivity of breastfeeding during the first month of life: effects on neonatal mortality and morbidity-a systematic review and meta-analysis. Matern Child Health J 2015;19(3):468-479

6. Mullany LC, Katz J, Li YM, et al. Breast-feeding patterns, time to initiation, and mortality risk among newborns in southern Nepal. J Nutr 2008;138(3):599-603

7. Edmond KM, Zandoh C, Quigley MA, Amenga-Etego S, Owusu-Agyei S, Kirkwood BR. Delayed breastfeeding initiation increases risk of neonatal mortality. Pediatrics 2006;117(3):e380-e386

8. Reading R. Effect of breastfeeding on infant and child mortality due to infectious diseases in less developed countries: a pooled analysis. Ambulatory Child Health 2000;6(2):133-134

9. Boccolini CS, de Carvalho ML, de Oliveira MIC, Pérez-Escamilla R. Breastfeeding during the first hour of life and neonatal mortality. Jornal de Pediatria 2013;89(2):131-136

10. Boucher O, Julvez J, Guxens M, et al. Association between breastfeeding duration and cognitive development, autistic traits and ADHD symptoms: a multicenter study in Spain. Pediatr Res 2017;81(3):434

11. Rodriguez-Lopez M, Osorio L, Acosta-Rojas R, et al. Influence of breastfeeding and postnatal nutrition on cardiovascular remodeling induced by fetal growth restriction. Pediatr Res 2016;79(11):100

12. Black RE, Victora CG, Walker SP, et al. Maternal and child undernutrition and overweight in lowincome and middle-income countries. The lancet 2013;382(9890):427-451

13. Rollins NC, Bhandari N, Hajeebhoy N, et al. Why invest, and what it will take to improve breastfeeding practices?. The Lancet 2016;387(10017):491-504

14. Clark SG, Bungum TJ. The benefits of breastfeeding: An introduction for health educators. California Journal of Health Promotion 2003;1(3):158

15. Elizabeth KE. Feeding of Young infants and Children in Exceptionally Difficult Circumstances (like, HIV and humanitarian emergencies): Solution Exchange for MCH Community Newsletter, Breastfeeding Month Special. 2008

16. World Health Organization. . Exclusive breastfeeding for six months best for babies everywhere.2011 2012

17. Ogbo FA, Page A, Idoko J, Claudio F, Agho KE. Diarrhoea and Suboptimal Feeding Practices in Nigeria: Evidence from the National Household Surveys. Paediatr Perinat Epidemiol 2016 
medRxiv preprint doi: https://doi.org/10.1101/19000497; this version posted July 8, 2019. The copyright holder for this preprint (which was not certified by peer review) is the author/funder, who has granted medRxiv a license to display the preprint in perpetuity. It is made available under a CC-BY-NC-ND 4.0 International license .

18. Lim SS, Vos T, Flaxman AD, et al. A comparative risk assessment of burden of disease and injury attributable to 67 risk factors and risk factor clusters in 21 regions, 1990-2010: a systematic analysis for the Global Burden of Disease Study 2010. The lancet 2012;380(9859):2224-2260

19. Begum K, Dewey KG. Impact of early initiation of exclusive breastfeeding on newborn deaths. 2010

20. Ayalew T, Tewabe T, Ayalew Y. Timely initiation of breastfeeding among first time mothers in Bahir Dar city, North West, Ethiopia, 2016. Pediatr Res 2019:1

21. Mekonnen Y, Tensou B, Telake DS, Degefie T, Bekele A. Neonatal mortality in Ethiopia: trends and determinants. BMC Public Health 2013;13(1):1

22. CSA I. Ethiopia demographic and health survey 2011. Addis Ababa, Ethiopia and Calverton, Maryland, USA: Central Statistical Agency and ICF International 2012

23. Aynalem YA, Habtewold TD, Shibabaw W, Yirga T, Dargie A. The magnitude of neonatal mortality and its predictors in Ethiopia: a systematic review and meta-analysis. bioRxiv 2019:626879

24. World Health Organization. Babies and mothers worldwide failed by lack of investment in breastfeeding. Saudi Med J 2017;38(9):974-975

25. Friedrich M. Early Initiation of Breastfeeding. JAMA 2018;320(11):1097-1097

26. Oakley L, Benova L, Macleod D, Lynch CA, Campbell OM. Early breastfeeding practices: Descriptive analysis of recent Demographic and Health Surveys. Maternal \& child nutrition 2018;14(2):e12535

27. Gebremedhin S. Core and optional infant and young child feeding indicators in Sub-Saharan Africa: a cross-sectional study. BMJ open 2019;9(2):bmjopen-2018-023238

28. Esteves TMB, Daumas RP, Oliveira, Maria Inês Couto de, Andrade, Carlos Augusto de Ferreira de, Leite IC. Factors associated to breastfeeding in the first hour of life: systematic review. Rev Saude Publica 2014;48(4):697-708

29. Macro O. Central Statistical Agency: Ethiopia Demographic and Health Survey 2005. ORC Macro, Calverton, Maryland, USA 2006

30. Habtewold T, Mohammed S, Endalamaw A, et al. Breast and complementary feeding in Ethiopia: new national evidence from systematic review and meta-analyses of studies in the past 10 years. Eur J Nutr 2018

31. Ogbo FA, Eastwood J, Page A, et al. The impact of sociodemographic and health-service factors on breast-feeding in sub-Saharan African countries with high diarrhoea mortality. Public Health Nutr 2017;20(17):3109-3119

32. Alzaheb RA. A Review of the Factors Associated With the Timely Initiation of Breastfeeding and Exclusive Breastfeeding in the Middle East. Clinical Medicine Insights: Pediatrics 2017; 11:1179556517748912

33. Benedict RK, Craig HC, Torlesse H, Stoltzfus RJ. Trends and predictors of optimal breastfeeding among children 0-23 months, South Asia: Analysis of national survey data. Maternal \& child nutrition 2018;14:e12698 
34. Sharma IK, Byrne A. Early initiation of breastfeeding: a systematic literature review of factors and barriers in South Asia. International breastfeeding journal 2016;11(1):17

35. Dhandapany G, Bethou A, Arunagirinathan A, Ananthakrishnan S. Antenatal counseling on breastfeeding-is it adequate? A descriptive study from Pondicherry, India. International Breastfeeding Journal 2008;3(1):1

36. Tarrant RC, Kearney JM. Session 1: Public health nutrition Breast-feeding practices in Ireland. Proc Nutr Soc 2008;67(04):371-380

37. Morhason-Bello IO, Adedokun BO, Ojengbede OA. Social support during child birth as a catalyst for early breastfeeding initiation for first-time Nigerian mothers. International breastfeeding journal 2009;4(1):1

38. Flacking R, Nyqvist KH, Ewald U. Effects of socioeconomic status on breastfeeding duration in mothers of preterm and term infants. Eur J Public Health 2007;17(6):579-584

39. Dearden K, Altaye M, Maza Id, et al. Determinants of optimal breast-feeding in peri-urban Guatemala City, Guatemala. Revista Panamericana de Salud Pública 2002;12(3):185-192

40. Habtewold TD, Sharew NT, Alemu SM. Effect of gender of new-born, antenatal care and postnatal care on breastfeeding practices in Ethiopia: Evidence from systematic review and metaanalysis of national studies. bioRxiv 2018:405605

41. Alemu SM, Alemu YM, Habtewold TD. Association of age and colostrum discarding with breastfeeding practice in Ethiopia: systematic review and meta-analyses. Public Health Nutr 2019:1-20

42. Gomez-Pomar E, Blubaugh R. The Baby Friendly Hospital Initiative and the ten steps for successful breastfeeding. a critical review of the literature. Journal of Perinatology 2018:1

43. Ethiopia Federal Ministry of Health. Ethiopian National strategy for Infant and Young Child Feeding (IYCF) 2004

44. Federal Democratic Republic of Ethiopia Ministry of Health. HSTP Health Sector Transformation Plan 2015/16-2019/20 (2008-2012 EFY) 2015

45. Assefa T, Samuel A, Argaw A, et al. Assessment of status of infant and young child feeding (IYCF) practice, policy and programs: Achievements and Gaps, in Ethiopia. European Journal of Nutrition \& Food Safety 2015:1085-1086

46. Federal Democratic Republic of Ethiopia. National Nutrition Program 2016-2020 2016

47. World Health Organization. Indicators for assessing infant and young child feeding practices: part 1: definitions: conclusions of a consensus meeting held 6-8 November 2007 in Washington DC, USA 2008

48. Moher D, Liberati A, Tetzlaff J, Altman DG, Prisma Group. Preferred reporting items for systematic reviews and meta-analyses: the PRISMA statement. PLoS med 2009;6(7):e1000097

49. Munn Z, Tufanaru C, Aromataris E. JBI's systematic reviews: data extraction and synthesis. Am J Nurs 2014;114(7):49-54

50. Peterson J, Welch V, Losos M, Tugwell P. . The Newcastle-Ottawa scale (NOS) for assessing the quality of nonrandomised studies in meta-analyses 2011 
medRxiv preprint doi: https://doi.org/10.1101/19000497; this version posted July 8, 2019. The copyright holder for this preprint (which was not certified by peer review) is the author/funder, who has granted medRxiv a license to display the preprint in perpetuity. It is made available under a CC-BY-NC-ND 4.0 International license .

51. Habtewold TD, Islam MA, Sharew NT, Mohammed SH, Birhanu MM, Tegegne BS. SystEmatic review and meta-aNAlysis of infanT and young child feeding Practices (ENAT-P) in Ethiopia: protocol. BMJ Open 2017;7(8):e017437-2017-017437

52. Egger M, Davey Smith G, Schneider M, Minder C. Bias in meta-analysis detected by a simple, graphical test. BMJ 1997;315(7109):629-634

53. Higgins J, Thompson SG. Quantifying heterogeneity in a meta-analysis. Stat Med 2002;21(11):1539-1558

54. Viechtbauer W. Conducting meta-analyses in R with the metafor package. Journal of statistical software 2010;36(3)

55. Tsumoto S, Hirano S. Formal Analysis of Leave-One-Out Methods Based on Decremental Sampling Scheme 2014;2:371-378

56. Demilew YM, Tafere TE, Abitew DB. Infant and young child feeding practice among mothers with 0-24 months old children in Slum areas of Bahir Dar City, Ethiopia. Int Breastfeed J 2017;12:26-0170117-x. eCollection 2017

57. Belachew A. Timely initiation of breastfeeding and associated factors among mothers of infants age 0-6 months old in Bahir Dar City, Northwest, Ethiopia, 2017: a community based cross-sectional study. International breastfeeding journal 2019;14(1):5

58. Adugna DT. Women's perception and risk factors for delayed initiation of breastfeeding in Arba Minch Zuria, Southern Ethiopia. Int Breastfeed J 2014;9:8-4358-9-8. eCollection 2014

59. Gultie T, Sebsibie G. Determinants of suboptimal breastfeeding practice in Debre Berhan town, Ethiopia: a cross sectional study. Int Breastfeed J 2016;11:5-016-0063-z. eCollection 2016

60. Derso T, Biks GA, Tariku A, et al. Correlates of early neonatal feeding practice in Dabat HDSS site, northwest Ethiopia. Int Breastfeed J 2017;12:25-017-0116-y. eCollection 2017

61. John JR, Mistry SK, Kebede G, Manohar N, Arora A. Determinants of early initiation of breastfeeding in Ethiopia: a population-based study using the 2016 demographic and health survey data. BMC pregnancy and childbirth 2019;19(1):69

62. Ekubay M, Berhe A, Yisma E. Initiation of breastfeeding within one hour of birth among mothers with infants younger than or equal to 6 months of age attending public health institutions in Addis Ababa, Ethiopia. International breastfeeding journal 2018;13(1):4

63. Alemayehu M, Abreha K, Yebyo H, Zemichael K, Gebremichael H. Factors associated with timely initiation and exclusive breast feeding among mothers of Axum town, Northern Ethiopia. Sci J Public Health 2014;2(5):394-401

64. Berhe H, Mekonnen B, Bayray A, Berhe H. Determinants of Breast feeding Practices Among Mothers Attending Public Health Facilities, Mekelle, Northern Ethiopia; A Cross Sectional Study. International Journal of Pharmaceutical Sciences and Research 2013;4(2):650

65. Beyene MG, Geda NR, Habtewold TD, Assen ZM. Early initiation of breastfeeding among mothers of children under the age of 24 months in Southern Ethiopia. Int Breastfeed J 2017;12:1-016-0096-3. eCollection 2016 
medRxiv preprint doi: https://doi.org/10.1101/19000497; this version posted July 8, 2019. The copyright holder for this preprint (which was not certified by peer review) is the author/funder, who has granted medRxiv a license to display the preprint in perpetuity.

It is made available under a CC-BY-NC-ND 4.0 International license .

66. Lakew Y, Tabar L, Haile D. Socio-medical determinants of timely breastfeeding initiation in Ethiopia: Evidence from the 2011 nation wide Demographic and Health Survey. Int Breastfeed J 2015;10:24-015-0050-9. eCollection 2015

67. Liben ML, Yesuf EM. Determinants of early initiation of breastfeeding in Amibara district, Northeastern Ethiopia: a community based cross-sectional study. Int Breastfeed J 2016;11:7-0160067-8. eCollection 2016

68. Setegn T, Gerbaba M, Belachew T. Determinants of timely initiation of breastfeeding among mothers in Goba Woreda, South East Ethiopia: a cross sectional study. BMC Public Health 2011;11:217-2458-11-217

69. Mekonen L, Seifu W, Shiferaw Z. Timely initiation of breastfeeding and associated factors among mothers of infants under 12 months in South Gondar zone, Amhara regional state, Ethiopia; 2013. International breastfeeding journal 2018;13(1):17

70. Tamiru D, Belachew T, Loha E, Mohammed S. Sub-optimal breastfeeding of infants during the first six months and associated factors in rural communities of Jimma Arjo Woreda, Southwest Ethiopia. BMC Public Health 2012;12:363-2458-12-363

71. Tamiru D, Tamrat M. Constraints to the optimal breastfeeding practices of breastfeeding mothers in the rural communities of Arba Minch Zuria Woreda, Ethiopia: A community-based, crosssectional study. S Afr J Clin Nutr 2015;28(3):134-139

72. Deregh. Breastfeeding practices among mothers of babies admitted to NICU, TAH, Addis Ababa, and factors influencing practice 2012

73. Woldemichael B, Kibie Y. Timely Initiation of Breastfeeding and Its Associated Factors among Mothers in Tiyo Woreda, Arsi Zone, Ethiopia: A Community-Based Cross Sectional Study. Clinics Mother Child Health 2016;13(221):2

74. Tamir. Assessment of optimal breastfeeding among workingand stay-at-home mothers in Bahir Dar town Amhararegional state, north west of Ethiopia 2010

75. Tariku A, Biks GA, Wassie MM, Worku AG, Yenit MK. Only half of the mothers practiced early initiation of breastfeeding in Northwest Ethiopia, 2015. BMC research notes 2017;10(1):501

76. Regassa N. Infant and child feeding practices among farming communities in Southern Ethiopia. Kontakt 2014;16(4):e215-e222

77. Musa Seid A. Vaginal Delivery and Maternal Knowledge on Correct Breastfeeding Initiation Time as Predictors of Early Breastfeeding Initiation: Lesson from a Community-Based Cross-Sectional Study. ISRN Epidemiology 2014;2014

78. Tilahun G, Degu G, Azale T, Tigabu A. Prevalence and associated factors of timely initiation of breastfeeding among mothers at Debre Berhan town, Ethiopia: a cross-sectional study. Int Breastfeed J 2016;11:27

79. Tewabe T. Timely initiation of breastfeeding and associated factors among mothers in Motta town, East Gojjam zone, Amhara regional state, Ethiopia, 2015: a cross-sectional study. BMC Pregnancy Childbirth 2016;16(1):314 
80. Bimerew A, Teshome M, Kassa GM. Prevalence of timely breastfeeding initiation and associated factors in Dembecha district, North West Ethiopia: a cross-sectional study. Int Breastfeed J

2016;11:28

81. Hailemariam TW, Adeba E, Sufa A. Predictors of early breastfeeding initiation among mothers of children under 24 months of age in rural part of West Ethiopia. BMC Public Health 2015;15:1076-

015-2420-z

82. Hoche S, Meshesha B, Wakgari N. Sub-optimal breastfeeding and its associated factors in rural communities of Hula District, southern Ethiopia: a cross-sectional study. Ethiopian journal of health sciences 2018;28(1):49-62

83. Acharya $P$, Khanal $V$. The effect of mother's educational status on early initiation of breastfeeding: further analysis of three consecutive Nepal Demographic and Health Surveys. BMC Public Health 2015;15(1):1069

84. Patel A, Banerjee A, Kaletwad A. Factors associated with prelacteal feeding and timely initiation of breastfeeding in hospital-delivered infants in India. Journal of Human Lactation 2013;29(4):572578

85. Senarath U, Dibley M, Agho K. Breastfeeding practices and associated factors among children under 24 months of age in Timor-Leste. Eur J Clin Nutr 2007;61(3):387

86. Silveira RBd, Albernaz E, Zuccheto LM. Fatores associados ao início da amamentação em uma cidade do sul do Brasil. Rev.bras.saúde mater.infant 2008;8(1):35-43

87. Esteves TMB, Daumas RP, Oliveira, Maria Inês Couto de, Andrade, Carlos Augusto de Ferreira de, Leite IC. Factors associated to breastfeeding in the first hour of life: systematic review. Rev Saude Publica 2014;48:697-708

88. Karkee R, Lee AH, Khanal V. Need factors for utilisation of institutional delivery services in Nepal: an analysis from Nepal Demographic and Health Survey, 2011. BMJ Open 2014;4(3):e004372-2013004372

89. Joshi C, Torvaldsen S, Hodgson R, Hayen A. Factors associated with the use and quality of antenatal care in Nepal: a population-based study using the demographic and health survey data. BMC pregnancy and childbirth 2014;14(1):94

90. Nawal D, Goli S. Birth preparedness and its effect on place of delivery and post-natal check-ups in Nepal. PloS one 2013;8(5):e60957

91. Gibson-Davis CM, Brooks-Gunn J. The association of couples' relationship status and quality with breastfeeding initiation. Journal of Marriage and Family 2007;69(5):1107-1117

92. Li R, Darling N, Maurice E, Barker L, Grummer-Strawn LM. Breastfeeding rates in the United States by characteristics of the child, mother, or family: the 2002 National Immunization Survey. Pediatrics 2005;115(1):e31-7

93. Seltzer JA. Families formed outside of marriage. Journal of marriage and family 2000;62(4):12471268

94. Mallik S, Dasgupta U, Naskar S, Sengupta D, Choudhury K, Bhattacharya S. Knowledge of breast feeding and timely initiation of it amongst post natal mothers: An experience from a baby friendly 
medRxiv preprint doi: https://doi.org/10.1101/19000497; this version posted July 8, 2019. The copyright holder for this preprint (which was not certified by peer review) is the author/funder, who has granted medRxiv a license to display the preprint in perpetuity. It is made available under a CC-BY-NC-ND 4.0 International license.

teaching hospital of a metropolitan city. IOSR Journal of Dental and Medical Sciences 2013;4(1):2530

95. Bich TH, Long TK, Hoa DP. Community-based father education intervention on breastfeeding practice-Results of a quasi-experimental study. Maternal \& child nutrition 2019;15:e12705

96. Li R, Scanlon KS, Serdula MK. The validity and reliability of maternal recall of breastfeeding practice. Nutr Rev 2005;63(4):103-110

97. Salasibew MM, Filteau S, Marchant T. A qualitative study exploring newborn care behaviours after home births in rural Ethiopia: implications for adoption of essential interventions for saving newborn lives. BMC Pregnancy Child birth 2014;14:412-014-0412-0 